\title{
Cholinergic deficits selectively boost cortical intratelencephalic control of the striatum in Huntington's disease
}

Dalton Surmeier ( $\nabla$ j-surmeier@northwestern.edu )

Northwestern University https://orcid.org/0000-0002-6376-5225

Tristano Pancani

Northwestern University

Michelle Day

Northwestern University Medical School

Tatiana Tkatch

Northwestern University

David Wokosin

Patricia Gonzalez Rodriguez

Feinberg School of Medicine/ Northwestern University https://orcid.org/0000-0002-2561-3984

Jyothisri Kondapalli

Northwestern University Medical School

Yu Chen

Northwestern University

Vahri Beaumont

CHDI Foundation

\section{Article}

Keywords: huntington's disease, corticostriatal dysfunction, cholinergic interneurons, intratelencephalic connections, pyramidal tract connections

Posted Date: November 23rd, 2021

DOI: https://doi.org/10.21203/rs.3.rs-1008365/v1

License: (c) (i) This work is licensed under a Creative Commons Attribution 4.0 International License.

Read Full License 


\section{Cholinergic deficits selectively boost cortical intratelencephalic control of the striatum in Huntington's disease}

Tristano Pancani ${ }^{1}$, Michelle Day ${ }^{1}$, Tatiana Tkatch ${ }^{1}$, David L. Wokosin ${ }^{1}$, Patricia Gonzalez-Rodriguez ${ }^{1}$, Jyothisri Kondapalli ${ }^{1}$, Yu Chen $^{1}$, Vahri Beaumont ${ }^{2}$ and D. James Surmeier ${ }^{1}$

${ }^{1}$ Department of Neuroscience, Feinberg School of Medicine, Northwestern University, Chicago, IL 60613 USA. ${ }^{2} \mathrm{CHDI}$ Management/CHDI Foundation, Suite 700, 6080 Center Drive, Los Angeles, CA 90045, USA.

Corresponding author:

D. James Surmeier, Ph.D.

Department of Neuroscience

Feinberg School of Medicine

Northwestern University

Chicago, IL 60613 USA

email: j-surmeier@northwestern.edu 


\section{Summary}

Huntington's disease (HD) is a progressive, neurodegenerative disease caused by a CAG triplet expansion in the huntingtin gene. Although corticostriatal dysfunction has long been implicated in HD, the determinants and pathway specificity of this pathophysiology remain a matter of speculation. To help fill this gap, the $z Q 175^{+/}$knockin mouse model of HD was studied using approaches that allowed optogenetic interrogation of intratelencephalic (IT) and pyramidal tract (PT) connections with principal striatal spiny projection neurons (SPNs). These studies revealed that the connectivity of IT, but not PT, neurons with direct and indirect pathway SPNs increased in early symptomatic zQ175 ${ }^{+/-}$HD mice. This enhancement was attributable to reduced inhibitory control of IT terminals by striatal cholinergic interneurons (Chls). Lowering mutant huntingtin selectively in Chls with a virally-delivered zinc finger repressor protein normalized striatal acetylcholine release and IT functional connectivity - revealing a novel node in the network underlying corticostriatal pathophysiology in HD. 


\section{Introduction}

Huntington's disease (HD) is a complex, neurodegenerative disease caused by a CAG expansion in the huntingtin gene. After an extended prodromal period, patients manifest progressive worsening of motor control and cognitive abilities ${ }^{1}$. Although mutant huntingtin ( $\mathrm{mHtt}$ ) is widely distributed, $\mathrm{HD}$ neuropathology is particularly prominent in the striatum ${ }^{2}$, a brain region belonging to the basal ganglia circuitry that regulates goal-directed actions, habit, and cognition ${ }^{3-5}$.

The principal neurons of the striatum are GABAergic spiny projection neurons (SPNs). The activity of SPNs is largely controlled by axospinous, excitatory glutamatergic synapses formed by projection neurons from the cerebral cortex. This innervation is derived from both intratelencephalic (IT) and pyramidal tract (PT) neurons ${ }^{6}$. IT neurons innervate both ipsilateral and contralateral striatum, whereas PT neurons exclusively innervate the ipsilateral striatum and more caudal structures $^{7-10}$. Although much remains to be learned about the information being transmitted by these pathways, PT signaling to the striatum is thought to convey information about ongoing motor execution, whereas IT signaling is related to motor planning, context and internal state ${ }^{10,11}$.

In HD models, a great deal of attention has been focused on the role of corticostriatal dysfunction in the evolution of striatal pathophysiology. Several lines of study have implicated deficits in corticostriatal signaling in HD arising from impaired transport and release of brain derived neurotrophic factor (BDNF) by cortical axons or activation of its postsynaptic receptor - tropomyosin receptor kinase $B(T r k B)^{12,13}$. Because TrkB signaling is necessary for activity-dependent potentiation of corticostriatal synapses and dendritic integration in $\mathrm{SPNs}^{14}$, these deficits could weaken the ability of the cerebral cortex to engage striatal circuits in movement control. Indeed, electrophysiological, and anatomical studies have revealed an apparent attenuation in the corticostriatal connectivity in HD models ${ }^{15-21}$.

However, there is a body of data that is difficult to reconcile with this model, particularly that having to do with striatal cholinergic interneurons (Chls). Although Chls are not lost in HD, there is compelling evidence that they are functionally compromised ${ }^{22-25}$. One of the major neuromodulatory targets of Chls are corticostriatal terminals, where acetylcholine (ACh) release acts through presynaptic muscarinic receptors to inhibit glutamate release ${ }^{26-28}$. Thus, a down-regulation in Chls function should result in an enhanced corticostriatal synaptic transmission, not a deficit as reported previously. A major complication in the interpretation of previous studies is their reliance upon electrical stimulation of tissue, typically in ex vivo brain slices. This approach not only fails to distinguish between IT and PT axons, but it also leads to activation of other axons, and cells, intrinsic to the striatum. To overcome this limitation, optogenetic approaches were used in the zQ175 ${ }^{+/-}$mouse model of $\mathrm{HD}^{29}$ to selectively interrogate IT and PT corticostriatal terminals, as well as to determine how Chls were modulating them. Surprisingly, these experiments revealed that functional synaptic connectivity of $M 1, M 2$ and cingulate cortex IT neurons with SPNs was elevated in the dorsal striatum of $z Q 175^{+/-}$mice. This change was traced to a deficit in activitydependent ACh release by Chls that resulted in weakening of M4 muscarinic receptor depression of IT terminal glutamate release. Reduction in $\mathrm{mHtt}$ in Chls with viral delivery of zinc finger proteins (ZFPs) ${ }^{30}$ normalized corticostriatal synaptic transmission but lowering $\mathrm{mHtt}$ in $\mathrm{M} 1$ cortex or in iSPNs did not.

\section{Results}

The connectivity of IT but not PT neurons with SPNs in was elevated in zQ175+/- mice

A major shortcoming of previous studies focused on corticostriatal connectivity in mouse models of HD has been the reliance upon electrical stimulation in ex vivo brain slices ${ }^{15,17-19,22,31-35}$. In the slice preparation, corticostriatal axons are invariably cut, limiting evoked responses with an electrical stimulus, and necessitating placement of the electrode close to or within the striatum; this placement allows current spread to the striatum itself, activating axons and neurons within this region. Not only does this make interpretation of changes in evoked responses problematic, it does not allow interrogation of specific corticostriatal networks. To overcome these limitations, optogenetic approaches were used in heterozygous zQ175 ${ }^{+/-}$mice to assess changes in the functional connectivity of IT and PT neurons with iSPNs and dSPNs in the dorsolateral striatum (DLS).

To measure IT synaptic transmission, an AAV vector carrying a synapsin-driven Chronos-GFP construct $(0.2 \mu$; AAV9.hSyn.Chronos-GFP; Fig. 1a, S1a) was injected into the M1 motor cortical region of 8-9 month old zQ175 ${ }^{+/}$and wildtype mice that expressed either tdTomato under control of the D1 receptor promoter, or eGFP under the D2 receptor promoter (allowing identification of dSPNs and iSPNs, respectively). To measure IT connectivity, whole-cell recordings with a $\mathrm{Cs}^{+}$-based internal solution were performed 20-30 days later from visually identified dSPNs and iSPNs in DLS contralateral to the AAV injection site. Unexpectedly, optically evoked excitatory postsynaptic currents (oEPSCs) from both zQ175 ${ }^{+/}$dSPNs and iSPNs were significantly larger than those in age-matched wildtype mice (Fig. 1b-e). A similar effect was seen when monitoring IT connectivity in SPNs from ipsilateral DLS using the TIx3(PL56)-Cre line (a mouse line expressing Cre in IT neurons) crossed into the $z Q 175^{+/}$line (Fig. S1b-e). Finally, the difference in IT connectivity was not evident in experiments with younger (2-3 monthold) mice (Fig. S1f-i). 
To determine whether this shift in connectivity was restricted to M1 IT projections, the functional connectivity of M2 motor cortex with DLS iSPNs was studied. Again, IT connectivity with iSPNs was elevated in slices from zQ175 ${ }^{+/-}$mice (Fig. S1j$\mathbf{m}$ ). To determine if the change was restricted to the DLS, the IT projection from the cingulate cortex (Cng.Ctx.) onto iSPNs in the dorsomedial striatum (DMS) was examined. Once more, IT connectivity with iSPNs was more robust in tissue from zQ175 ${ }^{+/-}$ mice (Fig. S1n-q).

In contrast to IT neurons, PT neurons project exclusively to the ipsilateral striatum, where they synapse on both dSPNs and iSPNs ${ }^{6}$. To assess the functional connectivity of PT neurons with SPNs, the Sim1-(KJ18)-Cre transgenic mouse line in which Cre-recombinase is expressed in layer 5 PT neurons (PT-cre) ${ }^{36}$ was used. Drd2-eGFP $x$ zQ175 $1 /-$ mice were crossed with the Sim1-KJ18 mice and then an AAV carrying a Cre-dependent, synapsin ChR2-eYFP expression construct was injected into the M1 motor cortex (Fig. 1f, S1r; $0.4 \mu$ l, AAV9.EF1a.DIO.ChR2-eYFP). Thirty days post-injection, oEPSCs were measured in DLS's dSPNs (eGFP negative) and neighboring iSPNs (eGFP positive) ipsilateral to the injection site. In contrast to the IT connectivity, the functional connectivity of PT neurons with dSPNs was significantly decreased (Fig. 1g,h) in slices from 8-9 month-old zQ175 ${ }^{+/-}$mice, but connectivity with iSPNs was not significantly changed (Fig. 1i,j).

\section{The change in IT functional connectivity was attributable to a presynaptic mechanism}

As a first step toward assessing the mechanism responsible for the elevation in IT responsiveness in zQ175 ${ }^{+/-}$SPNs, an estimate of the relative density of $\alpha$-amino-3-hydroxy-5-methyl-4-isoxazolepropionic acid receptors (AMPARs) and N-methyl$\mathrm{d}$-aspartate receptors (NMDARs) at IT synapses was generated. Postsynaptic long-term potentiation (LTP) of glutamatergic synapses is usually accompanied by an increase in the AMPAR to NMDAR ratio, as AMPAR are trafficked into the synapse ${ }^{37}$. Optogenetic methods, as described above, were used to selectively activate IT synapses on Cs ${ }^{+}$-filled iSPNs or dSPNs held at either $-70 \mathrm{mV}$ to estimate AMPAR currents or at $+40 \mathrm{mV}$ to estimate NMDAR currents (Fig. S2a). The ratio of these two currents was indistinguishable in SPNs from zQ175 ${ }^{+/-}$and wildtype mice (Fig. S2b).

If postsynaptic potentiation is set aside as an explanation for the change in IT responses, then there are two obvious alternatives: 1) the release probability at IT terminals increased or 2) the number of IT synapses increased. To explore the first possibility optogenetic strategies were employed using Chronos rather than ChR2 because of its ability to faithfully follow higher stimulation frequencies ${ }^{38}$. To determine if IT terminal glutamate release probability was altered in zQ175 ${ }^{+/-}$SPNs, contralateral M1 IT axons were optogenetically stimulated with a paired pulse protocol (100 msec inter-stimulus interval) while recording in whole cell voltage-clamp mode with a $\mathrm{Cs}^{+}$-based internal solution to maximize space clamp. The relative amplitude of the EPSC evoked in SPNs by the second of two optical stimuli was significantly smaller in zQ175 $5^{+/-}$SPNs (Fig. 2 a,b) - consistent with an elevation in the release probability of IT synapses.

To address the second possibility, subcellular channel-rhodopsin-assisted circuit mapping (sCRACM) was used to estimate the number of functional IT synapses on the dendrites of SPNs. In ex vivo brain slices from mice in which M1 neurons had been induced to express Chronos (as described in Fig. 1a), a blue laser beam (spot laser) was used to stimulate IT terminals synapsing on the spines of SPN dendrites in the contralateral striatum (Fig. 2c-e). These experiments were performed in the presence of tetrodotoxin (TTX) and 4-aminopyridine (4-AP) to block propagated activity. In addition, only distal dendrites were probed to avoid stimulating synapses above or below the plane of focus with the blue laser. Axo-spinous release of glutamate was monitored at the soma as time-locked oEPSCs (Fig. 2f) ${ }^{39}$. In the DLS from wildtype mice, 5-20\% of dSPNs and iSPNs spines had a detectable response to optogenetic activation of M1 IT terminals (Fig. $\mathbf{2 g}$ ). In DLS SPNs from zQ175 ${ }^{+/-}$mice, the proportion of spines having detectable responses was significantly greater (Fig. $\mathbf{2 g}$ ). The oEPSC amplitude distributions in zQ175 ${ }^{+/-}$were shifted towards larger amplitudes (Fig. 2h). Given the paired pulse data, these results are consistent with the proposition that the increased number of detectable IT synapses on SPNs dendrites reflects an increased probability of multivesicular release of glutamate and lack of postsynaptic receptor saturation at corticostriatal terminals ${ }^{26}$.

\section{Enhanced IT functional connectivity was attributable to diminished acety/choline release}

Several types of $\mathrm{G}_{\mathrm{i} / \mathrm{o}}$-coupled GPCRs inhibit glutamate release at corticostriatal synapses. Among the best described of these are $\mathrm{M} 2$-class muscarinic receptors ${ }^{26,27,40,41}$. Because studies of human brains and preclinical rodent models suggest that there is a striatal cholinergic deficit in $\mathrm{HD}^{23-25}$, our working hypothesis was that the enhanced functional connectivity of IT synapses was attributable to loss of pre-synaptic muscarinic modulation. To directly assess whether ACh release was altered in the $\mathrm{zQ} 175^{+/-}$striatum, a genetically-encoded optical sensor for $\mathrm{ACh}\left(\mathrm{AChSnFr}{ }^{42}\right)$ was packaged in an AAV and stereotaxically delivered in DLS (Fig. 3a,b). A month later, ex vivo slices were prepared for imaging. In agreement with the inferences of previous work, electrically evoked ACh release was significantly lower in striata from $z Q 175^{+/-}$mice than wildtype (Fig. 3c,d).

Although M2-class GPCRs are known to inhibit glutamate release from corticostriatal terminals, it is not known whether this modulation is specific to IT or PT terminals. To address this question, PT projections to the striatum were induced to express ChR2 by stereotaxic injection of AAV9.EF1a.DIO.ChR2-eYFP into M1 cortex of Drd2-eGFP mice crossed in the PT-Cre line (Fig. 4a). A month later, the effect of the muscarinic receptor agonist Oxo-M (10 $\mu \mathrm{M})$ on oEPSCs in ipsilateral SPNs in ex 
vivo slices was assessed in whole-cell voltage clamp recordings (Fig. $\mathbf{4 b}, \mathbf{c}$ ). The light intensity was adjusted to obtain oEPSCs that had peak amplitudes of about 100 pA to minimize clamping artifacts. Unexpectedly, Oxo-M had no effect on PT axons evoked oEPSCs in either dSPNs or iSPNs (Fig. 4b,c; Fig. S3a).

Next, the effects of mAChRs GPCRs on IT terminals was studied. To this end, AAV9.Syn.Chronos-GFP (0.2-0.3 $\mu$ l) was injected into the M1 cortex of Drd1-tdTomato mice (Fig. 4d; Fig. S3b). A month later, ex vivo slices were prepared and wholecell voltage clamp recordings were performed in contralateral dSPNs and presumed iSPNs (tdTomato-negative) as described above. In contrast to the experiments with PT axons, Oxo-M robustly reduced the peak amplitude of EPSC evoked by optical stimulation of IT axons. The modulation was observed in both dSPNs and iSPNs (Fig. 4e,f) and was accompanied by an increase in paired-pulse ratio (PPR) (Fig. 4g). Moreover, the modulation was persistent in both types of SPN (Fig. 4f).

To complement these pharmacological approaches, the ability of ACh release from Chls to mimic the muscarinic modulation of IT terminals was determined using a combination of chemogenetic and optogenetic approaches. To chemogenetically activate Chls, an AAV carrying a Cre-dependent, $5 \mathrm{HT} 3$ receptor-based 'pharmacologically selective actuator module' (PSAM-5HT3 ${ }^{43}$ ) expression construct was injected into the DLS of mice expressing Cre recombinase under control of the choline acetyltransferase promoter (ChAT-cre) and tdTomato under control of the Drd1 promoter. In the same mice, an AAV carrying synapsin-driven Chronos-GFP expression construct was stereotaxically injected into the contralateral M1 cortex, as described above (Fig. 5a,b). A month later, ex vivo slices were prepared and whole cell voltage clamp recordings obtained from dSPNs and presumed iSPNs as described above. PSAM-5HT3 activation was achieved by bath application of its cognate synthetic ligand (PSEM, pharmacologically selective effector module; $10 \mu \mathrm{M})^{43}$. In control recordings, PSEM robustly increased Chl spiking (Fig. 5c,d). When recording from SPNs, PSEM induced a reduction in optically evoked IT oEPSCs (Fig. 5e, f). The modulation was indistinguishable in dSPNs and iSPNs therefore the data was grouped. As with Oxo-M application, the modulation was long-lasting and persisted after washing out PSEM. As expected, this effect was also accompanied by an increase in PPR (Fig. 5g).

Presynaptic modulation of IT terminals was mediated by M4 muscarinic receptors

To better understand the differential modulation of IT and PT axon terminals by ACh, molecular approaches were used. AAV9.CAG.DIO-tdTomato was stereotaxically injected into the M1 cortex of either Sim1-(KJ18)-Cre (PT-specific Cre recombinase expression), or TIx3-(PL56)-Cre (IT-specific Cre recombinase expression) mice (Fig. 6a). A month later, M1 cortex was dissected out, dissociated, and then subjected to fluorescence-activated cell sorting (FACS). FACS isolated PT and IT neurons were then subjected to reverse transcription-quantitative polymerase chain reaction (RT-qPCR) analysis (Fig. S4a). This analysis revealed that M4 muscarinic receptor mRNA expression was roughly six-fold greater in IT than PT neurons (Fig. 6b). In contrast, the expression of M2 muscarinic receptor mRNA was relatively low and not different between the two cell types (Fig. 6b). To provide an independent confirmation of the functional significance of this difference, the M4 positive allosteric modulator VU0152100 (5 $\mathrm{\mu M})$ was co-applied with a sub-saturating concentration of the non-specific mAChRs agonist Oxo-M (100 nM) while monitoring oEPSCs amplitude in SPNs evoked by optogenetic stimulation of IT axons. While it had no effect on its own, VU0152100 roughly doubled the amplitude of the presynaptic Oxo-M modulation (Fig. 6c,d). This is consistent with the hypothesis that the modulation was mediated largely (if not exclusively) by M4Rs.

As mentioned above, one of the unexpected features of the IT modulation produced by cholinergic signaling was its persistence (Fig. 4f). Given that this effect was attributable to $\mathrm{G}_{\mathrm{i} / \mathrm{o}}$-coupled M4Rs, there are two signaling pathways that these

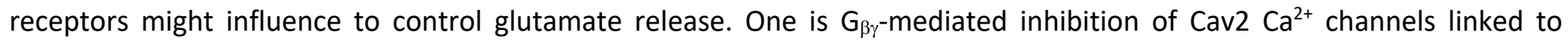
exocytosis ${ }^{44,45}$. The change in PPR produced by M4R activation is consistent with inhibition of active zone Cav2 $\mathrm{Ca}^{2+}$-channels ${ }^{44}$. But this membrane-delimited modulation typically rapidly reverses with agonist washout ${ }^{46}$. Another signaling linkage of $M 4 R s$ is $\mathrm{G}_{\alpha i}$-mediated inhibition of adenylyl cyclase (AC) and protein kinase A (PKA) (Fig. S4e $)^{44,47}$. Elevation of terminal cyclic adenosine monophosphate (CAMP) signaling is well known to enhance activity-dependent transmitter release ${ }^{48}$. To test for the involvement of cAMP signaling, the cell permeable cAMP analog 8-Br-cAMP was bath applied a few minutes before Oxo$\mathrm{M}$ while monitoring IT oEPSCs. Indeed, 8-Br-cAMP stimulation of PKA significantly reduced the duration of the Oxo-M modulation, but not its initial amplitude (Fig. 6e,f; S4d). A similar result was obtained with bath application of the AC activator forskolin (Fig. S4b,d). Finally, there is evidence that at least in dSPNs endogenous cholinergic signaling through M4R promote endocannabinoid-mediated long-term depression of corticostriatal glutamatergic synapses ${ }^{49}$, however, preincubation with the CB1 selective antagonist PF514273 did not block the Oxo-M effect (Fig. S4c,d). Taken together, these data suggest that M4R activation on IT terminals rapidly and transiently suppresses glutamate release through a $\mathrm{G}_{\beta \gamma}$ signaling pathway coupled to Cav2 $\mathrm{Ca}^{2+}$ channels, but also persistently diminishes glutamate release through $\mathrm{G}_{\alpha i}$-mediated inhibition of a constitutively active AC/PKA pathway (Fig. S4e) ${ }^{44,50}$.

Lowering $\mathrm{mHtt}$ in Chls, but not cortex, normalized IT connectivity in zQ175 ${ }^{+/-}$mice 
The data presented until this point suggests that $\mathrm{mHtt}$ in $\mathrm{zQ} 175^{+/-}$mice elevated glutamate release by IT synapses on SPNs either by attenuating M2/4R mediated presynaptic inhibition or by reducing ACh release by striatal Chls. To determine which mechanism was responsible, an AAV vector was used to deliver an expression construct for a zinc finger protein (ZFP) targeting the expanded CAG repeat of $\mathrm{mHtt}^{16}$. This ZFP leaves wildtype $\mathrm{Htt}$ unaltered, as previously described ${ }^{16}$. To assess off-target effects of ZFP expression, the ZFP that bound to mHtt mRNA (bZFP) was compared to a non-binding ZFP (nb-ZFP).

AAV9 vectors carrying synapsin promoter driven bZFP and nbZFP expression constructs were injected in the $M 1$ cortex of 6-7 month-old Drd2-eGFP zQ175+/- mice (Fig. S5a,b). Two months later, RT-qPCR analysis of M1 cortex confirmed that the bZFP, but not the nbZFP ${ }^{16}$, reduced mHtt mRNA abundance (Fig. S5c left); wildtype Htt mRNA abundance was unaffected by either ZFP (Fig. S5d). Additionally, there were no discernible alterations in the expression of mHtt or wildtype Htt contralateral to the bZFP injection site (Fig. S5c right). To assess the functional consequences of reducing cortical $\mathrm{mHtt}$, the $\mathrm{M} 1$ cortex was injected with AAV8 vector carrying a Chronos expression construct (Fig. 1) one month after the AAV9 ZFP vector (Fig. S5a,b). A month after injecting the Chronos vector, ex vivo brain slices were prepared and the functional connectivity of IT neurons with SPNs assessed as described above. Reducing $\mathrm{mHtt}$ in $\mathrm{M} 1$ cortex pyramidal neurons had no effect on IT evoked responses in SPNs (Fig. S5e-h).

Next, the same ZFP vectors were injected into the DLS (Fig. S6a,b), and the functional connectivity of IT pyramidal neurons with SPNs assessed (Fig. S6d-k). As with cortical targeting, the AAV reporter was broadly expressed by striatal neurons and the bZFP effectively lowered striatal (but not cortical) mHtt mRNA abundance (Fig. S6c) - leaving the wildtype Htt intact. In stark contrast to the effects of lowering $\mathrm{mHtt}$ in the cortex, lowering striatal $\mathrm{mHtt}$ normalized IT responses in SPNs (Fig. S6hk).

The question left unresolved by the experiments using a synapsin-driven ZFP expression construct was the striatal location of the $\mathrm{mHtt}$ responsible for the alteration in IT connectivity. Our working hypothesis was that Chl expression of mHtt was responsible. To test this hypothesis, two experiments were performed. First, $\mathrm{mHtt}$ was selectively lowered in $\mathrm{Chls}$ of $\mathrm{zQ} 175^{+/-}$ mice and then ACh release compared to that in age-matched control mice. To achieve cell-specific ZFP expression, ChAT-Cre $\mathrm{x} \mathrm{zQ175}{ }^{+-}$mice (as above) were injected with AAV9 vectors carrying Cre-dependent ZFP or ZFP control expression constructs (AAV9.DIO-bZFP-eGFP and AAV9.DIO-nbZFP-eGFP respectively). A month later, the AChSnFr AAV expression construct was delivered to the striatum (Fig. 7a,b) and a month later striatal ACh release was assessed as described above. Targeting the ZFP to Chls normalized ACh release in $\mathrm{zQ} 175^{+/-}$striata, while the control construct did not (Fig. $\mathbf{7 d - g}$ ). To provide an added control, cortical ACh release was measured; it was similar in slices from zQ175 ${ }^{+/}$and wildtype mice (Fig. S7), suggesting that basal forebrain cholinergic neurons (providing cholinergic innervation to the cortex) function is not altered at this stage in zQ175 ${ }^{+/}$. Next, the functional connectivity of IT neurons with SPNs was assessed after selectively reducing $\mathrm{mHtt}$ in $\mathrm{Ch} / \mathrm{s}$. If a reduction in cholinergic signaling was responsible for the corticostriatal pathophysiology in $z Q 175^{+/-}$mice, then normalizing ACh release by expressing ZFPs in Chls should correct it. To test this hypothesis, the striatum of ChAT-Cre $x$ zQ175 ${ }^{+/-}$mice was injected with an AAV9 vector carrying Cre-dependent ZFP and ZFP control expression constructs. An AAV8 Chronos expression construct was then introduced into the contralateral M1 cortex (Fig. 7h) as described above. As predicted, lowering $\mathrm{mHtt}$ selectively in Chls normalized IT responses in zQ175+/- SPNs (Fig. 8i-I).

To provide an additional test of the cellular specificity of the effect on IT connectivity with SPNs, the ZFP construct was expressed in iSPNs and then IT connectivity assessed. To this end, Adora2a-Cre mice (a mouse line expressing Cre in iSPNs neurons) were crossed into the $\mathrm{zQ} 175^{+/-}$line. The striatum of Adora2a-Cre $x \mathrm{zQ} 175^{+/-}$mice was then injected with the Credependent AAV9 ZFP vector and two months later IT connectivity assessed with optogenetic tools as described above (Fig. $\mathbf{S 8 a}, \mathbf{b})$. Lowering $\mathrm{mHtt}$ in iSPNs did not significantly alter the response to optogenetic stimulation of contralateral - M1 cortex - IT axons in $\mathrm{zQ} 175^{+/-}$(Fig. S8c,d).

\section{Discussion}

There are four conclusions that can be drawn from the experimental results presented. First, in the $\mathrm{zQ} 175^{+/-}$model of $\mathrm{HD}$, the functional connectivity of IT - but not PT - cortical pyramidal neurons with both iSPNs and dSPNs is increased. Second, this upregulation is attributable to de-depression of IT synaptic terminals. Third, this de-depression of IT synapses is due to a deficit in ACh release by $\mathrm{zQ} 175^{+/-} \mathrm{Chls}$, resulting in reduced presynaptic M4R signaling. Lastly, selectively lowering $\mathrm{mHtt}$ in zQ175 ${ }^{+/-}$Chls with a virally-delivered ZFP expression construct restores ACh release and normalizes IT synaptic connectivity. Taken together, these studies provide new insight into the network mechanisms underlying HD, as well as how cholinergic signaling regulates interhemispheric flow of information from the cerebral cortex to the striatum.

\section{Presynaptic M4Rs control the functional connectivity of IT, but not PT, cortical neurons with SPNs}

Striatal release of ACh by Chls has long been known to suppress glutamate release by corticostriatal terminals synapsing upon SPNs ${ }^{26-28}$. However, the selectivity and persistence of the modulation has not been appreciated. Using optogenetic approaches to selectively stimulate ipsilateral or contralateral IT inputs to SPNs, our studies demonstrate that M2-class 
mAChR signaling (particularly M4) potently inhibited glutamate release ${ }^{27}$. This inhibition was accompanied by an elevation in paired-pulse ratio, a hallmark of a presynaptic mechanism. In contrast, mAChR activation had no effect on PT axon terminals studied using the Sim1-KJ18 transgenic line of mice. This differential modulation was attributable to the preferential expression of M4Rs in IT neurons, as RT-PCR profiling revealed robust M4R mRNA expression in IT, but not PT, neurons and an M4R selective PAM enhanced the modulation.

As for a number of other $\mathrm{G}_{\mathrm{i} / \mathrm{o}}$-coupled GPCRs on corticostriatal terminals ${ }^{44,51,52}$, activation of M4Rs produced a persistent suppression of glutamate release. The persistence was not an artifact of applying exogenous mAChR ligands, as transient chemogenetic activation of Chls produced similar persistent suppression of synaptic transmission. Although the mechanisms responsible for the persistence of the modulation remain to be fully elucidated, the ability of targeted manipulation of $A C$ signaling to control this slow phase of the modulation is consistent with the proposition that transient M4R activation can have a long-lasting effect on constitutive PKA mediated enhancement of activity-dependent exocytosis ${ }^{44}$. This feature of the M4R modulation, and that of other $\mathrm{G}_{\mathrm{i} / \mathrm{o}}$-coupled GPCRs, merits additional study.

The enhancement of IT connectivity in $\mathrm{zQ175^{+/ }}$ mice is attributable to a deficit in ChI ACh release

In agreement with inferences drawn from previous studies, evoked ACh release from $z Q 175^{+/}$Chls was significantly lower than wildtype Chls. ACh release was measured using a novel optical probe (AChSnFr) expressed in the striatum using an AAV vector $^{42}$. The deficit in $\mathrm{Chl}$ signaling provided a simple explanation for the enhanced functional connectivity of IT neurons with both $\mathrm{zQ} 175^{+/}$iSPNs and dSPNs. Although our release experiments focused on the DLS, the corticostriatal enhancement was evident at terminals from M1, M2 and CgCtx in both DLS and DMS, suggesting it was not limited to DLS and M1. Although the cholinergic innervation of the striatum is not limited to $\mathrm{Chls}^{53}$, their causal role in the alteration in IT connectivity was cemented by the observation that knocking down mHtt broadly in striatal neurons or selectively in Chls normalized the

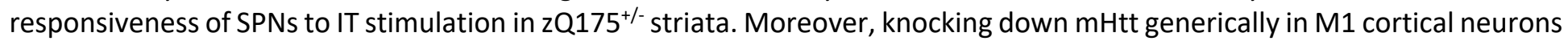
or selectively in iSPNs did not affect IT synaptic function.

A presynaptic locus for the change in IT connectivity in $\mathrm{zQ} 175^{+/-}$mice was supported by three lines of evidence. First, the ratio of currents through AMPA and NMDA receptors was indistinguishable at IT synapses on zQ175 ${ }^{+/}$and wildtype SPNs, suggesting that postsynaptic potentiation was not a factor. Second, using Chronos to reliably stimulate IT axons repetitively, it was found that the release probability at IT synaptic terminals was elevated in ex vivo brain slices from zQ75 ${ }^{+/-}$mice, as predicted if there was a loss presynaptic M4R signaling. Third, the number of dendritic spines having a detectable response to presynaptic optical stimulation of IT terminals was significantly greater in $\mathrm{zQ} 175^{+/-}$SPNs. This change was attributable to a shift in the amplitude distribution of postsynaptic responses, moving a significant number of spines above our experimental detection threshold. Given that the postsynaptic complement of AMPA receptors had not changed and glutamate release at corticostriatal synapses is multi-vesicular and does not saturate postsynaptic glutamate receptors ${ }^{26}$, this shift can be ascribed to removal of a persistent presynaptic inhibition of glutamate release - as predicted. It is also worth noting that enhanced glutamate release at $\mathrm{zQ} 175^{+/}$IT terminals may provide an additional explanation for the reported engagement of extrasynaptic glutamate receptors in HD models ${ }^{54}$.

How can our results be reconciled with previous reports of impaired corticostriatal synaptic transmission in HD models? One factor to consider is age of the model. The shift in IT connectivity was developmentally regulated, appearing only about the time $\mathrm{zQ} 175^{+/-}$mice begin to manifest deficits ${ }^{55}$. Another methodological factor was our use of optogenetic approaches to selectively activate IT axons, whereas previous studies have relied upon electrical stimulation that engages not only IT and PT corticostriatal axons, but also other glutamatergic axons (e.g., thalamostriatal), as well as neurons intrinsic to the striatum, like Chls. This clearly complicates the interpretation of these previous results. In fact, electrical stimulation could bias SPN responses toward those dependent upon PT synaptic terminals, as PT axons should be larger and have a lower threshold for electrical stimulation ${ }^{56}$; moreover, as PT terminals appeared to be dis-facilitated, possibly by loss of nAChR activation, this methodological difference could be a key to reconciling studies. Also, inadvertent electrical stimulation of Chls could have increased ACh release in HD tissue, masking the baseline enhancement of IT connectivity.

Another factor to consider is the extent to which postsynaptic mechanisms were engaged in assessing functional connectivity. In our experiments, neurons were loaded with $\mathrm{Cs}^{+}$and voltage-clamped to minimize the contribution of postsynaptic mechanisms, but this was not the case in a number of previous studies of HD models ${ }^{57}$. Previous work by our group has shown that not only is postsynaptic LTP at glutamatergic synapses compromised in zQ175 ${ }^{+/}$iSPNs, but that dendritic excitability also is reduced ${ }^{14,16}$. The deficit in cholinergic signaling described here should work together with these cell autonomous deficits induced by $\mathrm{mHtt}$, as $\mathrm{mAChR}$ signaling enhances the dendritic excitability of iSPNs and promotes LTP induction ${ }^{28,58}$. Thus, it is reasonable to infer that, at least in iSPNs, spiking induced by electrical stimulation in ex vivo brain slices from HD models could be less robust, despite the dis-inhibition of IT terminals.

Functional implications of altered Chl regulation of corticostriatal transmission in HD 
How does the recognition that $\mathrm{mHtt}$ impairs $\mathrm{Chl}$ ACh release and enhances IT connectivity with SPNs impact our understanding of HD? Several lines of study suggest that Chls are important to set-shifting; that is, changing action selection with changing environmental circumstances or contingencies ${ }^{28,59}$. The mechanisms mediating set-shifting are far from being completely understood, but it does appear that it involves changing the balance in excitability of iSPNs and dSPNs toward iSPNs and cessation of on-going thoughts and actions ${ }^{28}$. As noted above, the cell-autonomous effects of mHtt on the excitability of iSPNs and the ability to potentiate corticostriatal glutamatergic synaptic transmission should work together with the deficit in Chl signaling to create a 'perfect storm' in the indirect pathway, disrupting the ability to suppress unwanted actions.

What is less clear is how biasing SPN inputs toward IT and away from PT pathways fits into this scenario. It is thought that PT pathways carry information about ongoing actions, whereas IT pathways carry information about internal state, sensory context, and motor planning ${ }^{10,11}$. Thus, transient $\mathrm{Chl}$ suppression of IT synapses could serve to diminish the influence of previously reinforced, contextual cues and planned actions on striatal ensembles, allowing new striatal ensembles to emerge that could drive alternative, more adaptive actions. A presynaptic mechanism with a slow time course could be a particularly effective means of 'disrupting' striatal ensembles without completely erasing memories stored in postsynaptic circuitry. Regardless, the mechanisms for set-shifting and adaptive behavior should be impaired in HD. Indeed, a hallmark of HD are perseverative, obsessive behaviors ${ }^{60}$. This inference is of considerable translational value as ZFP-mediated reduction in $\mathrm{mHtt}$ expression in symptomatic mice was able to normalize ACh release and the functional connectivity of IT neurons with SPNs. While selective targeting of Chls was effective, the fact that $\mathrm{mHtt}$ drives other, cell-autonomous forms of pathology, particularly in iSPNs, suggests that broadly targeting $\mathrm{mHtt}$ in the striatum to include SPN, Chls and astrocytes ${ }^{16,61}$ is likely to be the most effective therapeutic path forward for HD patients. 


\section{Bibliography}

1 Paulson, H. L. \& Albin, R. L. in Neurobiology of Huntington's Disease: Applications to Drug Discovery Frontiers in Neuroscience (eds D. C. Lo \& R. E. Hughes) (2011).

2 Rub, U. et al. Huntington's disease (HD): the neuropathology of a multisystem neurodegenerative disorder of the human brain. Brain Pathol 26, 726-740, doi:10.1111/bpa.12426 (2016).

3 Lipton, D. M., Gonzales, B. J. \& Citri, A. Dorsal Striatal Circuits for Habits, Compulsions and Addictions. Front Syst Neurosci 13, 28, doi:10.3389/fnsys.2019.00028 (2019).

$4 \quad$ Gerfen, C. R. \& Surmeier, D. J. Modulation of striatal projection systems by dopamine. Annu Rev Neurosci 34, 441466, doi:10.1146/annurev-neuro-061010-113641 (2011).

Aarts, E., van Holstein, M. \& Cools, R. Striatal Dopamine and the Interface between Motivation and Cognition. Front Psychol 2, 163, doi:10.3389/fpsyg.2011.00163 (2011). Kress, G. J. et al. Convergent Shepherd, G. M. Corticostriatal connectivity and its role in disease. Nat Rev Neurosci 14, 278-291, doi:10.1038/nrn3469 (2013).

Cowan, R. L. \& Wilson, C. J. Spontaneous firing patterns and axonal projections of single corticostriatal neurons in the rat medial agranular cortex. J Neurophysiol 71, 17-32, doi:10.1152/jn.1994.71.1.17 (1994).

Wilson, C. J. Morphology and synaptic connections of crossed corticostriatal neurons in the rat. J Comp Neurol 263, 567-580, doi:10.1002/cne.902630408 (1987).

Reiner, A., Hart, N. M., Lei, W. \& Deng, Y. Corticostriatal projection neurons - dichotomous types and dichotomous functions. Front Neuroanat 4, 142, doi:10.3389/fnana.2010.00142 (2010). Li, N., Chen, T. W., Guo, Z. V., Gerfen, C. R. \& Svoboda, K. A motor cortex circuit for motor planning and movement. Nature 519, 51-56, doi:10.1038/nature14178 (2015).

Gauthier, L. R. et al. Huntingtin controls neurotrophic support and survival of neurons by enhancing BDNF vesicular transport along microtubules. Cell 118, 127-138, doi:10.1016/j.cell.2004.06.018 (2004).

13 Zuccato, C. \& Cattaneo, E. Role of brain-derived neurotrophic factor in Huntington's disease. Prog Neurobiol 81, 294330, doi:10.1016/j.pneurobio.2007.01.003 (2007).

Plotkin, J. L. et al. Impaired TrkB receptor signaling underlies corticostriatal dysfunction in Huntington's disease. Neuron 83, 178-188, doi:10.1016/j.neuron.2014.05.032 (2014).

Andre, V. M. et al. Differential electrophysiological changes in striatal output neurons in Huntington's disease. J Neurosci 31, 1170-1182, doi:10.1523/JNEUROSCI.3539-10.2011 (2011). neurons. Elife 8, doi:10.7554/eLife.40818 (2019).

Cepeda, C. et al. Transient and progressive electrophysiological alterations in the corticostriatal pathway in a mouse model of Huntington's disease. J Neurosci 23, 961-969 (2003).

Joshi, P. R. et al. Age-dependent alterations of corticostriatal activity in the YAC128 mouse model of Huntington disease. J Neurosci 29, 2414-2427, doi:10.1523/JNEUROSCI.5687-08.2009 (2009).

Pancani, T. et al. Allosteric activation of M4 muscarinic receptors improve behavioral and physiological alterations in early symptomatic YAC128 mice. Proc Natl Acad Sci U S A 112, 14078-14083, doi:10.1073/pnas.1512812112 (2015).

Plotkin, J. L. \& Surmeier, D. J. Corticostriatal synaptic adaptations in Huntington's disease. Curr Opin Neurobio/ 33, 5362, doi:10.1016/j.conb.2015.01.020 (2015).

21 Macdonald, V. \& Halliday, G. Pyramidal cell loss in motor cortices in Huntington's disease. Neurobiol Dis 10, 378-386, doi:10.1006/nbdi.2002.0528 (2002). doi:10.3389/fnins.2020.00082 (2020). striatal neurons in Huntington's disease. Brain Res 411, 162-166, doi:10.1016/0006-8993(87)90694-9 (1987).

Deng, Y. P. \& Reiner, A. Cholinergic interneurons in the Q140 knockin mouse model of Huntington's disease: Reductions in dendritic branching and thalamostriatal input. J Comp Neurol 524, 3518-3529, doi:10.1002/cne.24013 (2016).

Reiner, A. \& Deng, Y. P. Disrupted striatal neuron inputs and outputs in Huntington's disease. CNS Neurosci Ther 24, 250-280, doi:10.1111/cns.12844 (2018). 
Higley, M. J., Soler-Llavina, G. J. \& Sabatini, B. L. Cholinergic modulation of multivesicular release regulates striatal synaptic potency and integration. Nat Neurosci 12, 1121-1128, doi:10.1038/nn.2368 (2009).

Pancani, T. et al. M4 mAChR-mediated modulation of glutamatergic transmission at corticostriatal synapses. ACS Chem Neurosci 5, 318-324, doi:10.1021/cn500003z (2014).

Ding, J. B., Guzman, J. N., Peterson, J. D., Goldberg, J. A. \& Surmeier, D. J. Thalamic gating of corticostriatal signaling by cholinergic interneurons. Neuron 67, 294-307, doi:10.1016/j.neuron.2010.06.017 (2010).

Menalled, L. B. et al. Comprehensive behavioral and molecular characterization of a new knock-in mouse model of Huntington's disease: zQ175. PLoS One 7, e49838, doi:10.1371/journal.pone.0049838 (2012).

Zeitler, B. et al. Allele-selective transcriptional repression of mutant HTT for the treatment of Huntington's disease. Nat Med 25, 1131-1142, doi:10.1038/s41591-019-0478-3 (2019).

Klapstein, G. J. et al. Electrophysiological and morphological changes in striatal spiny neurons in R6/2 Huntington's disease transgenic mice. J Neurophysiol 86, 2667-2677, doi:10.1152/jn.2001.86.6.2667 (2001).

Rebec, G. V., Conroy, S. K. \& Barton, S. J. Hyperactive striatal neurons in symptomatic Huntington R6/2 mice: variations with behavioral state and repeated ascorbate treatment. Neuroscience 137, 327-336, doi:10.1016/j.neuroscience.2005.08.062 (2006).

Indersmitten, T., Tran, C. H., Cepeda, C. \& Levine, M. S. Altered excitatory and inhibitory inputs to striatal mediumsized spiny neurons and cortical pyramidal neurons in the Q175 mouse model of Huntington's disease. J Neurophysiol 113, 2953-2966, doi:10.1152/jn.01056.2014 (2015).

Miller, B. R., Walker, A. G., Barton, S. J. \& Rebec, G. V. Dysregulated Neuronal Activity Patterns Implicate Corticostriatal Circuit Dysfunction in Multiple Rodent Models of Huntington's Disease. Front Syst Neurosci 5, 26, doi:10.3389/fnsys.2011.00026 (2011).

Rothe, T. et al. Pathological gamma oscillations, impaired dopamine release, synapse loss and reduced dynamic range of unitary glutamatergic synaptic transmission in the striatum of hypokinetic Q175 Huntington mice. Neuroscience 311, 519-538, doi:10.1016/j.neuroscience.2015.10.039 (2015).

Gerfen, C. R., Paletzki, R. \& Heintz, N. GENSAT BAC cre-recombinase driver lines to study the functional organization of cerebral cortical and basal ganglia circuits. Neuron 80, 1368-1383, doi:10.1016/j.neuron.2013.10.016 (2013). Kauer, J. A., Malenka, R. C. \& Nicoll, R. A. A persistent postsynaptic modification mediates long-term potentiation in the hippocampus. Neuron 1, 911-917, doi:10.1016/0896-6273(88)90148-1 (1988).

Klapoetke, N. C. et al. Independent optical excitation of distinct neural populations. Nat Methods 11, 338-346, doi:10.1038/nmeth.2836 (2014).

Fieblinger, T. et al. Cell type-specific plasticity of striatal projection neurons in parkinsonism and L-DOPA-induced dyskinesia. Nat Commun 5, 5316, doi:10.1038/ncomms6316 (2014).

Malenka, R. C. \& Kocsis, J. D. Presynaptic actions of carbachol and adenosine on corticostriatal synaptic transmission studied in vitro. J Neurosci 8, 3750-3756 (1988).

Pakhotin, P. \& Bracci, E. Cholinergic interneurons control the excitatory input to the striatum. J Neurosci 27, 391-400, doi:10.1523/JNEUROSCI.3709-06.2007 (2007).

Borden, P. M. et al. A fast genetically encoded fluorescent sensor for faithful <em>in vivo</em> acetylcholine detection in mice, fish, worms and flies. bioRxiv, 2020.2002.2007.939504, doi:10.1101/2020.02.07.939504 (2020). Magnus, C. J. et al. Chemical and genetic engineering of selective ion channel-ligand interactions. Science 333, 12921296, doi:10.1126/science.1206606 (2011).

Atwood, B. K., Lovinger, D. M. \& Mathur, B. N. Presynaptic long-term depression mediated by Gi/o-coupled receptors. Trends Neurosci 37, 663-673, doi:10.1016/j.tins.2014.07.010 (2014).

Herlitze, S. et al. Modulation of Ca2+ channels by G-protein beta gamma subunits. Nature 380, 258-262, doi:10.1038/380258a0 (1996).

Bourinet, E., Soong, T. W., Stea, A. \& Snutch, T. P. Determinants of the G protein-dependent opioid modulation of neuronal calcium channels. Proc Natl Acad Sci U S A 93, 1486-1491, doi:10.1073/pnas.93.4.1486 (1996). 85, 1303-1342, doi:10.1152/physrev.00001.2005 (2005).

Ariel, P., Hoppa, M. B. \& Ryan, T. A. Intrinsic variability in Pv, RRP size, Ca(2+) channel repertoire, and presynaptic potentiation in individual synaptic boutons. Front Synaptic Neurosci 4, 9, doi:10.3389/fnsyn.2012.00009 (2012). Dyskinesia. Neuron 88, 762-773, doi:10.1016/j.neuron.2015.10.039 (2015). 
Fritz, B. M., Yin, F. \& Atwood, B. K. Input-selective adenosine A1 receptor-mediated synaptic depression of excitatory transmission in dorsal striatum. Sci Rep 11, 6345, doi:10.1038/s41598-021-85513-x (2021).

52 Monday, H. R., Younts, T. J. \& Castillo, P. E. Long-Term Plasticity of Neurotransmitter Release: Emerging Mechanisms and Contributions to Brain Function and Disease. Annu Rev Neurosci 41, 299-322, doi:10.1146/annurev-neuro080317-062155 (2018).

53 Dautan, D. et al. A major external source of cholinergic innervation of the striatum and nucleus accumbens originates in the brainstem. J Neurosci 34, 4509-4518, doi:10.1523/JNEUROSCI.5071-13.2014 (2014).

54 Milnerwood, A. J. et al. Early increase in extrasynaptic NMDA receptor signaling and expression contributes to phenotype onset in Huntington's disease mice. Neuron 65, 178-190, doi:10.1016/j.neuron.2010.01.008 (2010).

55 Southwell, A. L. et al. An enhanced Q175 knock-in mouse model of Huntington disease with higher mutant huntingtin levels and accelerated disease phenotypes. Hum Mol Genet 25, 3654-3675, doi:10.1093/hmg/ddw212 (2016).

Reiner, A., Jiao, Y., Del Mar, N., Laverghetta, A. V. \& Lei, W. L. Differential morphology of pyramidal tract-type and intratelencephalically projecting-type corticostriatal neurons and their intrastriatal terminals in rats. J Comp Neurol 457, 420-440, doi:10.1002/cne.10541 (2003).

57 Beaumont, V. et al. Phosphodiesterase 10A Inhibition Improves Cortico-Basal Ganglia Function in Huntington's Disease Models. Neuron 92, 1220-1237, doi:10.1016/j.neuron.2016.10.064 (2016).

58 Calabresi, P., Centonze, D., Gubellini, P. \& Bernardi, G. Activation of M1-like muscarinic receptors is required for the induction of corticostriatal LTP. Neuropharmacology 38, 323-326, doi:10.1016/s0028-3908(98)00199-3 (1999).

59 Thorn, C. A. \& Graybiel, A. M. Pausing to regroup: thalamic gating of cortico-basal ganglia networks. Neuron 67, 175178, doi:10.1016/j.neuron.2010.07.010 (2010).

60 van Duijn, E. et al. Neuropsychiatric symptoms in a European Huntington's disease cohort (REGISTRY). J Neurol Neurosurg Psychiatry 85, 1411-1418, doi:10.1136/jnnp-2013-307343 (2014).

61 Diaz-Castro, B., Gangwani, M. R., Yu, X., Coppola, G. \& Khakh, B. S. Astrocyte molecular signatures in Huntington's disease. Sci Transl Med 11, doi:10.1126/scitranslmed.aaw8546 (2019). 


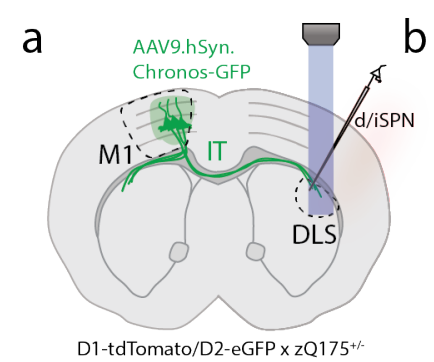

$f$

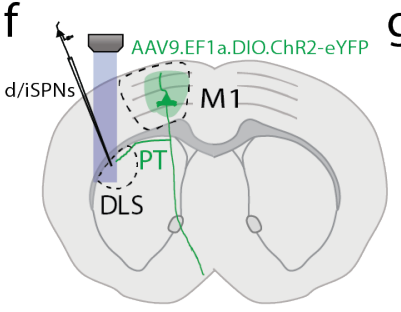

D2-eGFP x PT-cre x zQ175

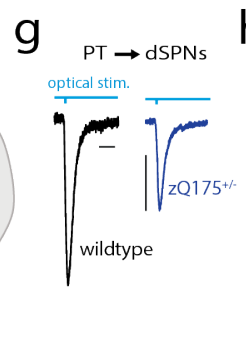

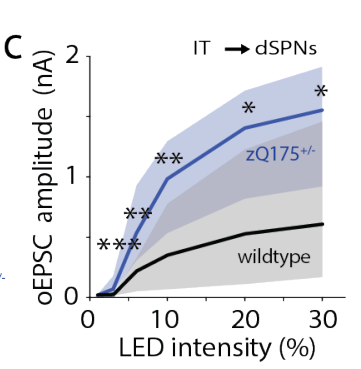

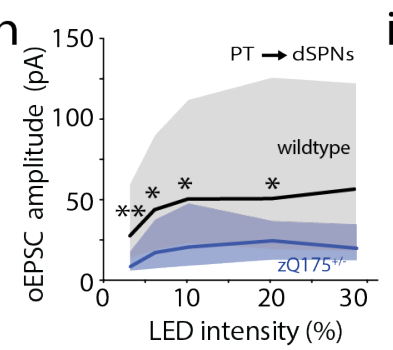

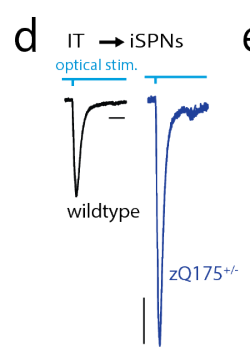
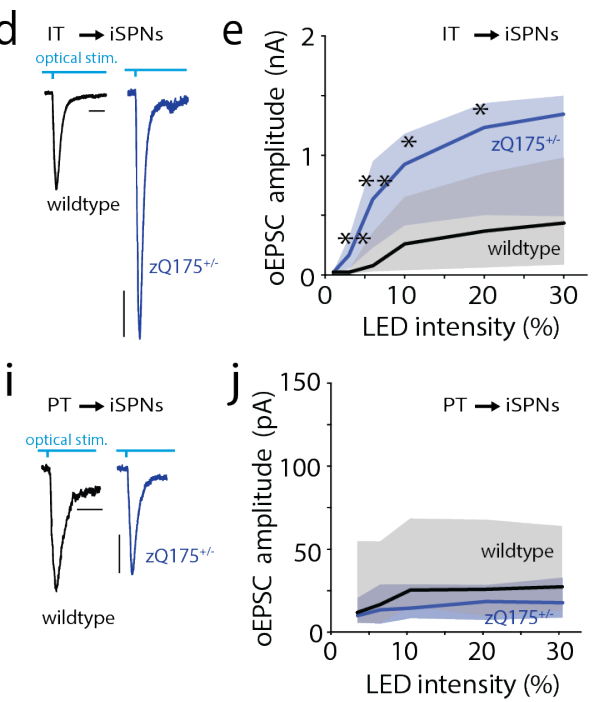

Figure 1 Increased connectivity at IT-SPNs synapses with no change or loss of PT connectivity in $\mathrm{zQ}^{175^{+/-}}$mice. (a) Schematics of AAV9.hSyn.Chronos-GFP injected in primary motor cortex (M1) in a double transgenic mouse obtained crossing D1-tdTomato or D2-eGFP with $\mathrm{zQ} 175^{+/-}$mice. Whole-cell recording from SPNs in dorsolateral striatum (DLS) in the contralateral hemisphere. (b, d) Representative traces of optically evoked EPSCs (oEPSC) recorded from dSPNs $\mathbf{b}$ and iSPNs d (Scale bars: $10 \mathrm{~ms}$ and 200pA). (c, e) Graphs showing increased oEPSCs amplitude when plotted against LED intensity in zQ175 ${ }^{+/-}$mice compared to wildtype controls. Input/output curves (I/O) in dSPNs (c; wildtype ( $\mathrm{N}=5$ mice; $\mathrm{n}=10$ neurons) vs. zQ175 ${ }^{+/-}(\mathrm{N}=4 ; \mathrm{n}=17)$; p-value (LED Intensity, \% of max): n.s. (1 \%), ***0.0004 (3\%), **0.0047 (6 \%), **0.0470 (10\%), $* 0.0110(20 \%), * 0.0350(30 \%))$, and iSPNs (e; wildtype ( $N=5 ; n=11)$ vs. $z Q 175^{+/-}(\mathrm{N}=4 ; n=15) ; p$-value (LED Intensity, \% of $\max )$ : n.s. (1 \%), ${ }^{* *} 0.0054$ (3\%), ${ }^{* *} 0.0036$ (6 \%), *0.0163 (10\%), *0.0475 (20\%), n.s. 0.0623 (30 \%)). (f) Schematic representation of the AAV9.EF1a.DIO.ChR2-GFP injection in a triple transgenic mouse obtained crossing D2-eGFP with PTcre and $z Q 175^{+/-}$mice (see also Figure S1n). Whole-cell recording performed in ipsilateral DLS. (g, i) Representative traces of oEPSCs recorded from SPNs (Scale bars: $10 \mathrm{~ms}$ and $10 \mathrm{pA}$ for dSPNs; $10 \mathrm{~ms}$ and $20 \mathrm{pA}$ iSPNs). (h, j) Graphs summarizing eEPSCs peak amplitude data plotted against LED intensity from I/O curves recorded in dSPNs (wildtype ( $N=3 ; n=14)$, zQ175 ${ }^{+-}(\mathrm{N}=7 ; \mathrm{n}=14)$, p-value (Stim. Intensity, \% of max): **0.0580 (3 \%), *0.0186 (6\%), *0.0182 (10\%), *0.0241 (20\%), $0.0556(30 \%)$ ), and iSPNs (wildtype $(N=3 ; n=7), z Q 175^{+-}(N=7 ; n=20), p$-value (Stim. Intensity, \% of max): n.s. (1\%), n.s. (3\%), n.s. (6\%), n.s. (10\%), n.s. $(20 \%)$, n.s. $(30 \%))$. (c, e, h, j) line represents median; shaded area includes $\mathrm{min} / \mathrm{max}$. Mann-Whitney non-parametric tests. 


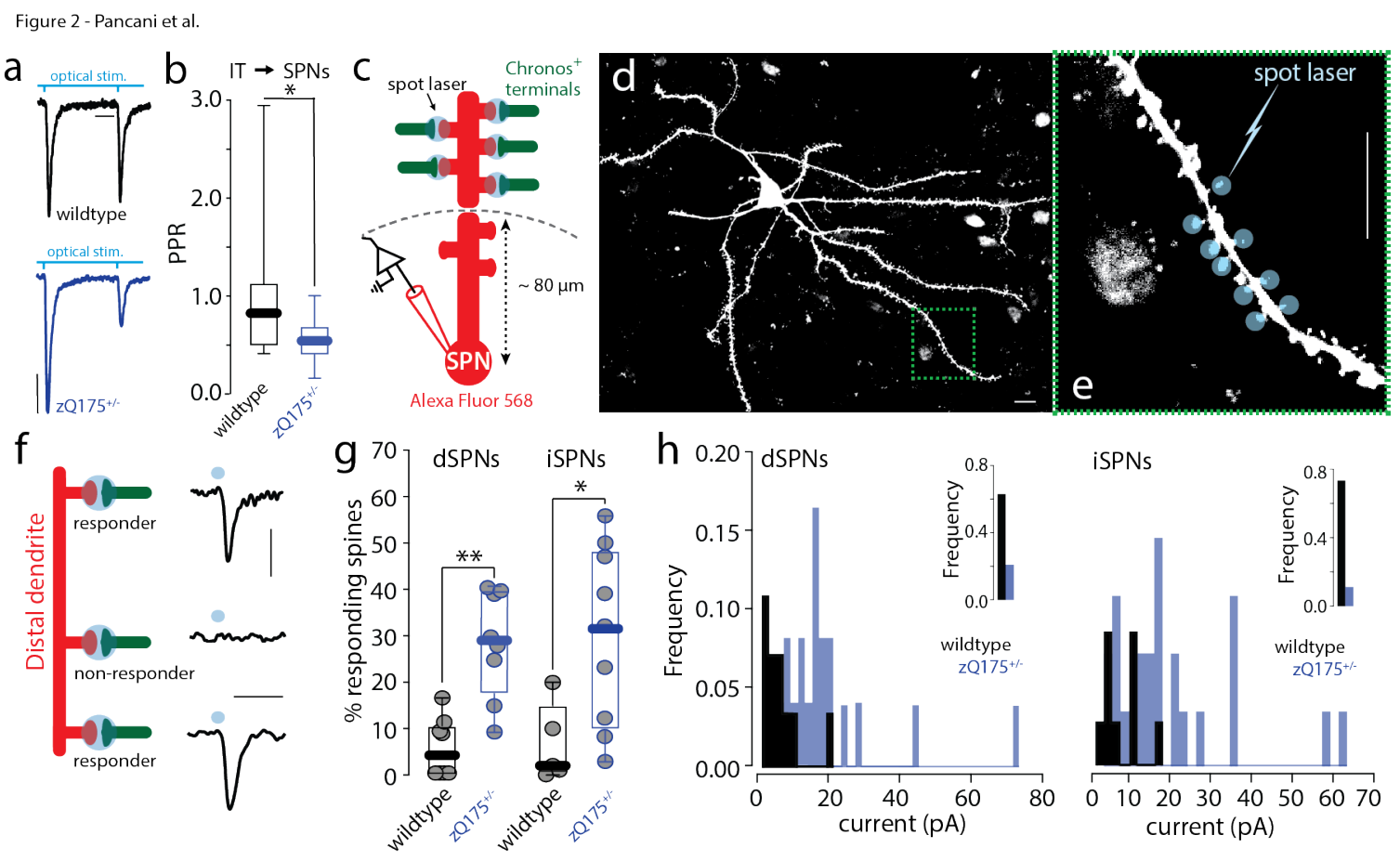

Figure 2 Presynaptic changes in IT axo-spinous synapses in $\mathrm{zQ}_{175^{+/}}$. (a) Sample oEPSCs evoked by a paired-pulse stimulation of IT terminals (Scale bars: $25 \mathrm{~ms}$ and 50 pA; see Figure 1a for AAV9.hSyn.Chronos-GFP stereotaxic delivery). (b) Boxplot summary showing reduced PPRs (oEPSC $2 /$ oEPSC $_{1}$ ) in $2 Q 175^{+/-}$compared to wildtype controls (wildtype $\mathrm{N}=5$; $n=16$ and $z Q 175^{+/} N=7 ; n=26 ;{ }^{*} p=0.011$ ). (c) Representation of a dendrite (proximal/distal sections shown) emanating from an SPN patched and dialyzed with Alexa Fluor 568. In green, contralateral IT terminals expressing chronos. Blue circles depict the spot laser beam $(\varnothing \sim 1 \mu \mathrm{m}, 475 \mathrm{~nm}, 1 \mathrm{~ms}$ duration) used for focal spine stimulation. (d) Representative dSPN filled with Alexa 568 (Scale bar: $10 \mu \mathrm{m}$ ). (e) Parfocal distal dendrite. Each spine is being sequentially stimulated with one laser pulse (Scale bar: $10 \mu \mathrm{m}$ ). (f) Traces of somatic oEPSCs evoked by a single light pulse delivered on each visible parfocal spine. Figure shows spines generating a somatic OEPSC (responder) or not (non-responder spine; Scale bars: 20 $\mathrm{ms}$ and $20 \mathrm{pA}$ ). (g) Boxplots summarizing the results. Significant increase in the number of active axo-spinous synapses in the distal compartment of both dSPNs and iSPNs in $\mathrm{zQ} 175^{+/}$animals (boxplots represent median and interquartile range, whiskers $\mathrm{min} / \mathrm{max}$ value; dSPNs wildtype $(\mathrm{N}=7 ; \mathrm{n}=8), \mathrm{zQ175^{+/- }}(\mathrm{N}=6 ; \mathrm{n}=8),{ }^{*} \mathrm{p}=0.001$ in a Mann-Whitney non-parametric test; iSPNs wildtype $(N=4 ; n=5), z Q 175^{+/-}(N=4 ; n=9), * p=0.019$ in a Mann-Whitney non-parametric test. (h) histogram of oEPSC amplitudes in wildtype compared to $z Q 175^{+-}$in dSPNs (left) and iSPNs (right). Inserts represents the frequency no response $(0 \mathrm{pA})$.

Figure 3 - Pancani et al.
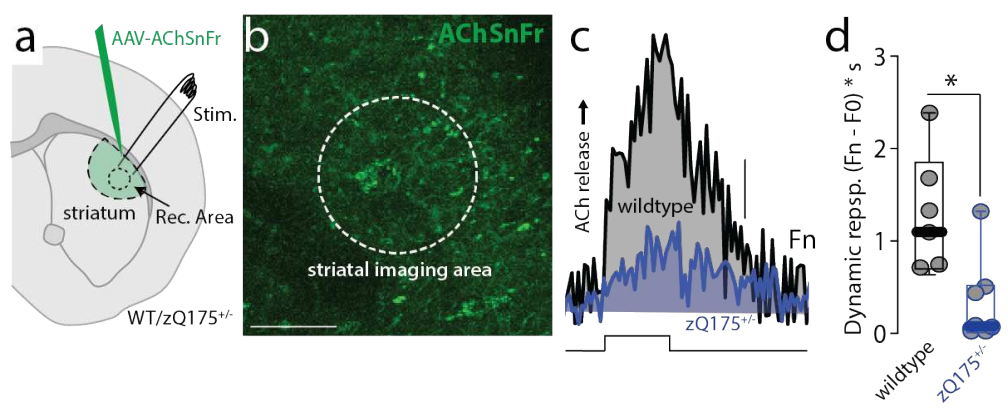

Figure 3 Reduced evoked ACh release in DLS in $\mathrm{ZQ175}^{+/-}$mice. (a) Diagram showing AAV-AChSnFr stereotaxically injected in DLS. The imaging area and bipolar electrical stimulator are indicated. (b) DLS section showing the striatal neuropil expressing AAV-AChSnFr and the imaging area (Scale bar: $50 \mu \mathrm{m}$ ). (c) Representative traces showing a decrease in evoked $\mathrm{ACh}$ released in $\mathrm{zQ} 175^{+/-}$compared to wildtype. Scale bar: 0.05 Normalized Fluorescence (Fn). Electrical stimulation (step) consisted of $20-1 \mathrm{~ms}$ stimuli, of $1 \mathrm{~mA}$ delivered at $2 \mathrm{~Hz}$. (d) Boxplot summarizing the data from wildtype ( $N=3 ; n=6$ 
slices), $\mathrm{zQ} 175^{+/-}(\mathrm{N}=4 ; \mathrm{n}=7),{ }^{* *} \mathrm{p}=0.0082$ Mann-Whitney non-parametric test. Shown is the Dynamic response [(Fn - F0) * s].

Figure 4 - Pancani et al.
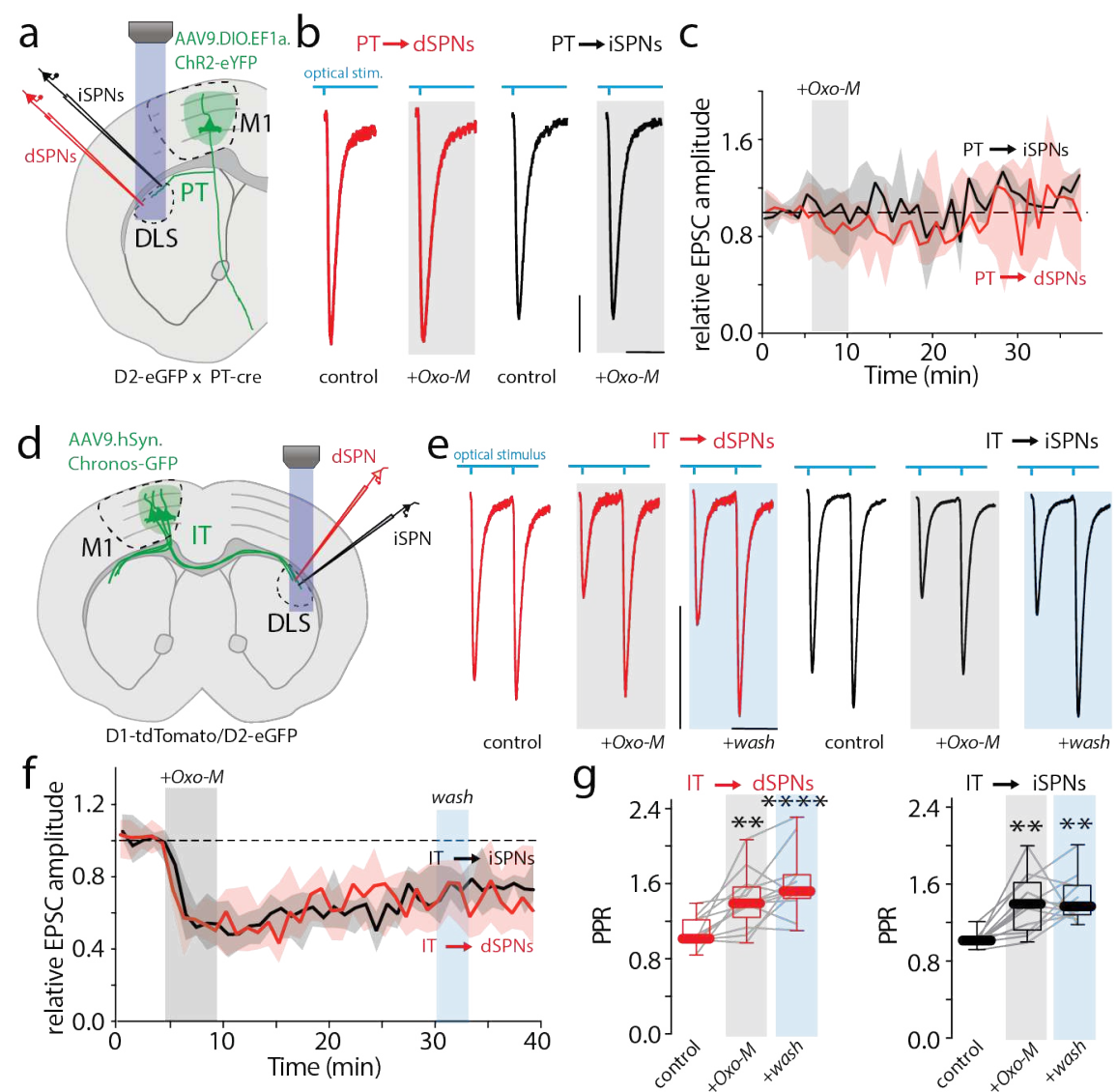

Figure 4 Muscarinic activation modulates IT corticostriatal transmission, but not PT. (a) Schematic of AAV9.EF1a.DIO.ChR2-GFP delivered in M1 of D2-eGFP crossed with PT-cre mice. Whole-cell recordings were obtained in DLS from ipsilateral SPNs. (b) Representative oEPSC traces from dSPNs (red) and iSPNs (black) obtained by stimulating PT terminals. Traces acquired at baseline (control), and during Oxo-M (10 $\mu \mathrm{M})$ perfusion (Scale bars: $10 \mathrm{~ms}$ and $50 \mathrm{pA})$. (c) Time-series of oEPSC peak amplitudes normalized to control and plotted over time. No effect of five minutes application of Oxo-M (10 $\mu \mathrm{M})$ on oEPSC amplitude. (d) Schematics of AAV9.hSyn.Chronos-GFP delivered in M1 of D1-tdTomato or D2eGFP mice. Whole-cell recordings were obtained from SPNs in contralateral DLS. (e) Representative oEPSC traces obtained by stimulating IT terminals and recorded from dSPNs (red) and iSPNs (black) before, during and after (wash) Oxo-M (10 $\mu \mathrm{M}$ ) perfusion (Scale bars: $50 \mathrm{~ms}$ and $50 \mathrm{pA}$ ). (f) Five minutes Oxo-M application induces a similar and long-lasting reduction of oEPSC amplitude in both dSPNs and iSPNs. (g) Significant increase in PPR ratio during Oxo-M perfusion and during the wash period. In dSPNs, control vs. Oxo-M $(\mathrm{N}=4 ; \mathrm{n}=13),{ }^{*} \mathrm{p}=0.0650$; control vs. wash $(\mathrm{N}=4 ; \mathrm{n}=13)$, $* * * * p<0.0001$. In iSPNs, control vs. Oxo-M $(N=4 ; n=13),{ }^{* *} p=0.0019 ;$ control vs. wash $(N=3 ; n=8),{ }^{* *} p=0.0090$ in a KruskalWallis test with Dunn's multiple comparisons. 


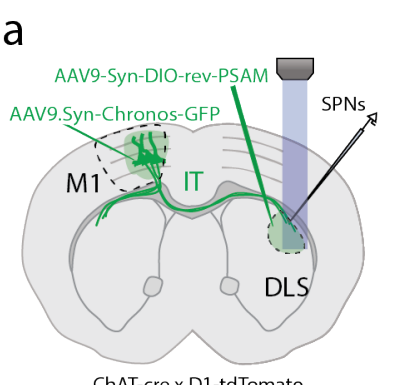

ChAT-cre x D1-tdTomato

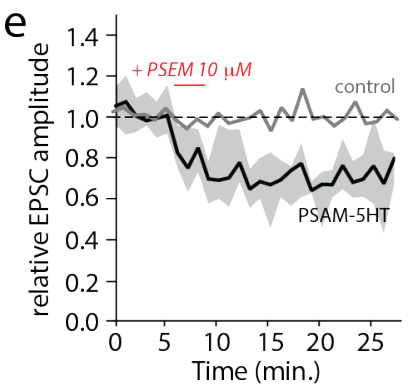

b
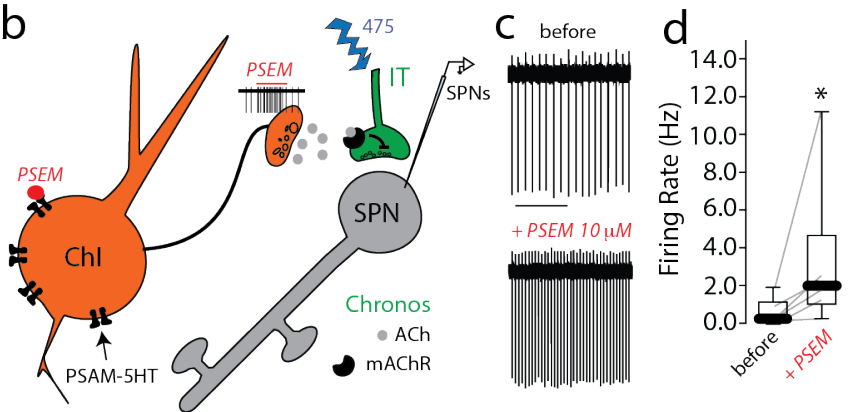

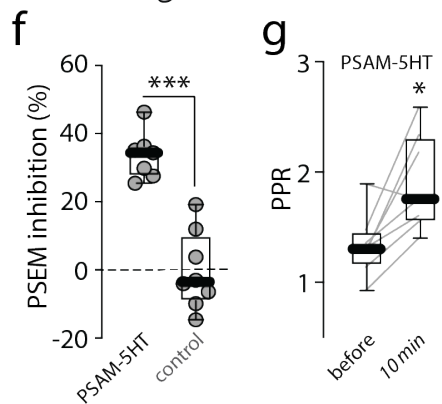

Figure 5 Endogenous acetylcholine release inhibits IT corticostriatal transmission. (a) We stereotaxically injected an AAV vector carrying $5 \mathrm{HT}$ receptor-based pharmacologically selective actuator module (PSAM-5HT) - associated to a Credependent expression construct (AAV.DIO.PSAM.5HT3-GFP) - in DLS of ChAT-cre mice. In the same mice, we injected an AAV vector carrying the AAV9.hSyn.Chronos-GFP construct in M1 contralateral to the PSAM-injected striatum. Thirty days post injection, we performed whole-cell recordings in SPNs from the striatum expressing PSAM-5HT. (b) Cartoon depicting pharmacologically selective effector module (PSEM) activation of PSAM-5HT-expressing ChI. Presynaptic activation of mAChRs reduces glutamate release from IT terminals. (c,d) Strong increase in firing rate of Chls expressing PSAM achieved by bath application of PSEM $(10 \mu \mathrm{M})$. (c) representative traces obtained in cell-attached configuration (Scale bar: 4 seconds). (d) boxplot summary of the results ( ${ }^{*} p=0.0312$, in a Wilcoxon paired test; $N=3, n=6$ pairs). (e) Time-series data describing the prolonged oEPSC inhibition obtained with three minutes bath application of PSEM (10 $\mu \mathrm{M})$ when recording from SPNs (control $\mathrm{N}=4, \mathrm{n}=9$; PSAM-5HT-injected mice $\mathrm{N}=4, \mathrm{n}=9$ ). (f) Inhibitory effect of PSEM on oEPSCs amplitude in slices from mice injected with AAV.DIO.PSAM.5HT3-GFP compared to non-injected mice (control). The inhibitory effect of PSEM is present only in PSAM-5HT injected mice (at 20-23 minutes after PSEM application: PSAM-5HT $N=3, n=7$; control $\mathrm{N}=4, \mathrm{n}=8 ;{ }^{* *} \mathrm{p}=0.0003$ in a Mann-Whitney non-parametric test). (f) PSEM stimulation of Chls increases oEPSCs PPR ratio recorded in SPNs compared to baseline control (before; ${ }^{*} \mathrm{p}=0.0156$, in a Wilcoxon paired test; $\mathrm{n}=8$ pairs). 


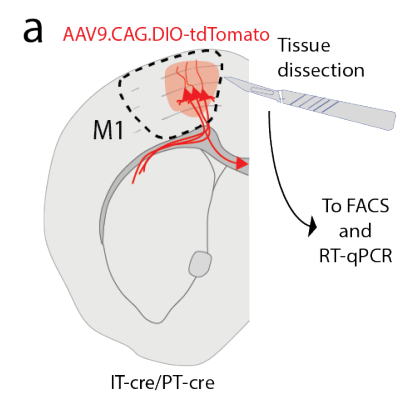

$C_{\text {onters }}$

IT-cre/PT-cre
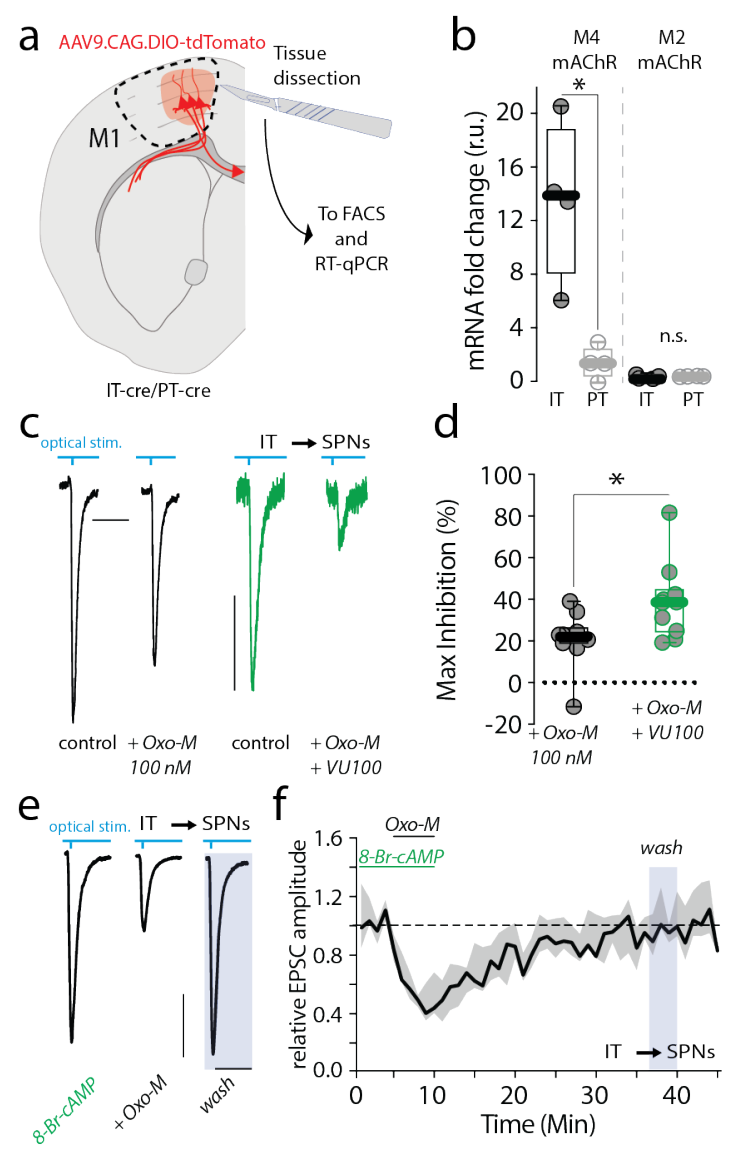

Figure 6 M4 modulation of IT corticostriatal transmission. (a) Schematic of AAV9.CAG.DIO-tdTomato stereotaxically delivered in $\mathrm{M} 1$ in Sim1(KJ18)-Cre (PT-cre) and Tlx3(PL56)-Cre (IT-cre) mice. M1 tdTomato positive are enzymatically separated, sorted with Fluorescence-activated cell sorting (FACS), and analyzed (See also Figure S4a and Materials and Methods). (b) Boxplot showing that M4 muscarinic receptor (mAChR) mRNA in IT neurons is significantly higher compared to PT (IT N=4 animals, PT N=4 animals; ${ }^{*} \mathrm{p}=0.013$ in a Mann-Whitney non-parametric test). No difference in $\mathrm{M} 2$ expression. (c) Representative oEPSC traces recorded from SPNs and obtained by stimulating IT terminals as described in Figure 1a (Scale bars: $50 \mathrm{~ms}$ and $100 \mathrm{pA}$ ). Traces acquired before (control), and during five minutes bath application of a sub-maximal concentration of Oxo-M (100 nM). In the experiments with the M4 PAM VU0152100 (green, VU100, $5 \mu M$ ), VU100 was pre-applied for at least 15-20 minutes before adding Oxo-M. (d) Boxplots showing that bath application of VU100 potentiates the inhibitory effects of Oxo-M (100 nM) on IT transmission ( ${ }^{*} p=0.0185$ in a Mann-Whitney non-parametric test, $\mathrm{N}=3 ; \mathrm{n}=10$ for both groups). (e) Representative oEPSC traces recorded from SPNs and obtained by stimulating IT terminals (Scale bars: $20 \mathrm{~ms}$ and $50 \mathrm{pA}$ ). 15-20 min pre-incubation with the cell-permeable cAMP analog 8-Br-cAMP blocked the long-term depression (LTD) obtained with five min. application of Oxo-M (10 $\mu \mathrm{M})$. (f) Time-series data describing the effects of pre-incubating the slices with 8-Br-cAMP on the chemically induced, muscarinic-dependent LTD (see also Figure S4). No significant effect of 8-Br-cAMP was seen on the acute inhibition. 

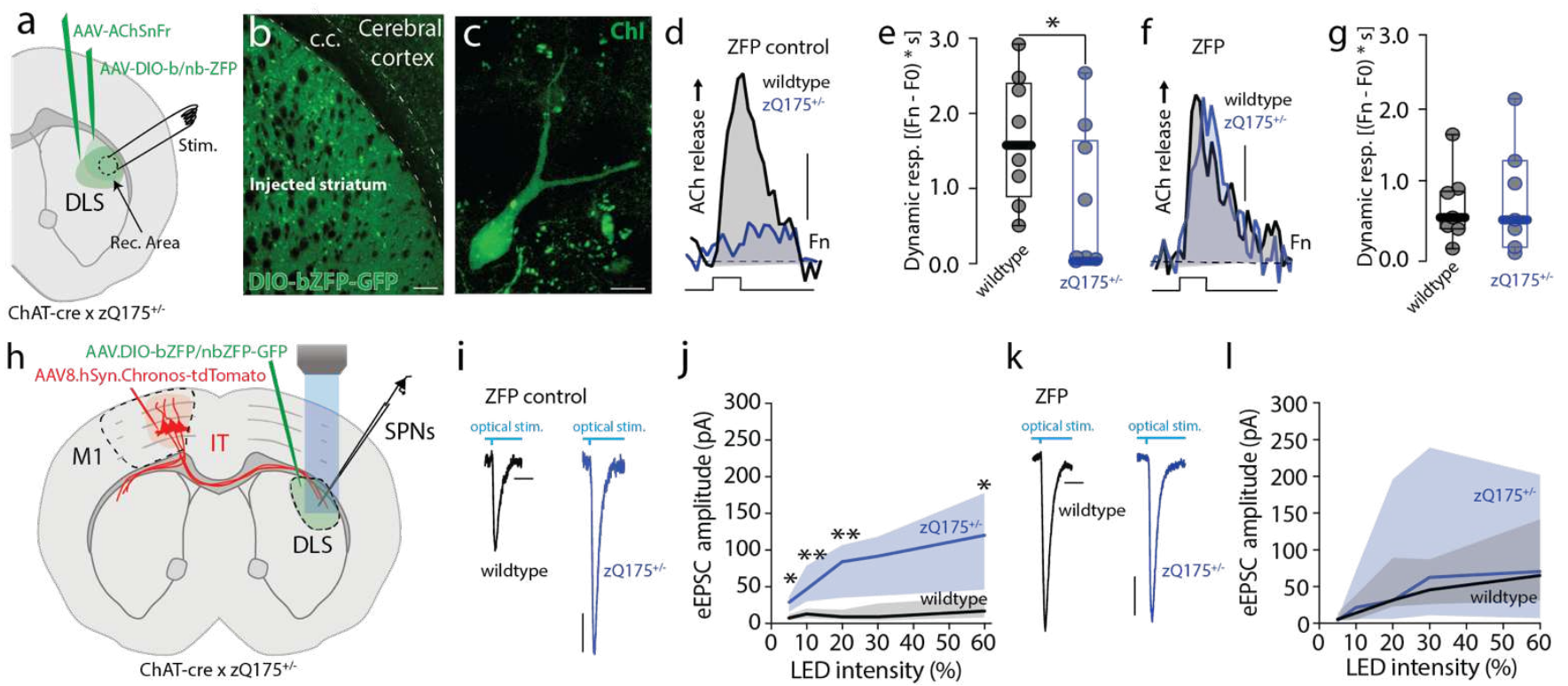

Figure 7 Lowering $\mathrm{mHtt}$ in Chls normalizes ACh release and IT connectivity in $\mathbf{z Q 1 7 5}^{+/-}$mice. (a) Schematic of AAV.AChSnFr and AAV.DIO.ZFP/ZFP control-eGFP viral constructs injected in DLS of ChAT-cre $x$ zQ175 ${ }^{+/-}$mice. (b) Confocal image of DLS (Scale bar: $100 \mu \mathrm{m}$ ) showing high expression of the AAV.DIO-bZFP-eGFP construct in ChAT-cre neurons c (Scale bar: $20 \mu \mathrm{m}$ ). (d, e) Mice expressing ZFP control construct in Chls display reduced ACh release. Representative traces are shown in $\mathbf{d}$ (Scale bar: 0.1$)$, while e shows summary bar-graph - wildtype of the dynamic response $(N=4, n=8$ slices), zQ175 ${ }^{+/-}(\mathrm{N}=4, \mathrm{n}=10),{ }^{*} \mathrm{p}=0.04343$. (f, $\left.\mathbf{g}\right)$ ZFP expression in Chls normalizes evoked ACh release. Representative traces in $\mathbf{f}$ (Scale bar: 0.1) while g shows summary data - wildtype ( $N=3, n=7$ slices), $z Q 175^{+-}(N=3, n=7$ slices), $p>0.9999$. All tests are Mann-Whitney non-parametric tests. (h) Schematic depicting the experimental paradigm used for virus delivery and SPN whole-cell recording in ChAT-cre $x$ zQ175 ${ }^{+/}$. (i) Expression of the ZFP control construct selectively in ChAT+ neurons in DLS did not normalize IT corticostriatal transmission. Representative traces of oEPSCs elicited optically stimulating IT axons, and (j) $\mathrm{l} / \mathrm{O}$ curves obtained in wildtype compared to $\mathrm{zQ} 175^{+/-}$- wildtype ( $\left.N=4, n=14\right), \mathrm{zQ} 175^{+/-}(\mathrm{N}=3, \mathrm{n}=8) \mathrm{p}$-value (Stim. Intensity, \% of max): ${ }^{*} 0.0016(5 \%),{ }^{* *} 0.0063$ (10\%), ${ }^{* *} 0.0081(30 \%),{ }^{*} 0.0081(60 \%)$ in a multiple Mann-Whitney nonparametric test. $(\mathbf{k}, \mathbf{l})$ Expression of the ZFP construct in ChAT-cre neurons normalized IT transmission in $\mathrm{zQ} 175^{+/}$- compared to wildtype. wildtype $(\mathrm{N}=4, \mathrm{n}=13), \mathrm{zQ175}{ }^{+-}(\mathrm{n}=11) \mathrm{p}$-value (Stim. Intensity, \% of max): 0.5309 (5\%), $0.6084(10 \%), 0.8646$ (20\%), 0.8646 (30\%), 0.9546 (60\%) in a multiple Mann-Whitney non-parametric test. (Scale bar in i and k: $10 \mathrm{~ms} 50 \mathrm{pA})$. 


\section{Supplementary figures}

Figure S1 - Pancani et al.
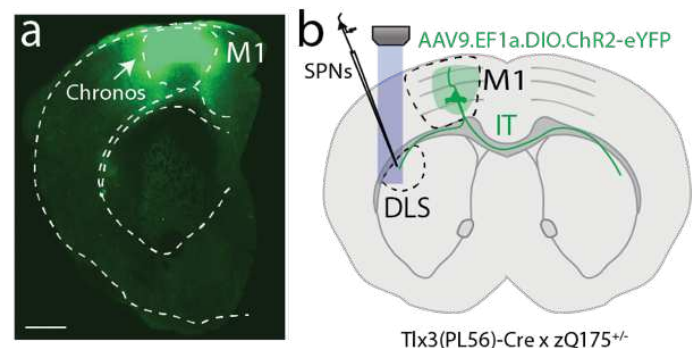

f $\underset{\substack{2-3 \text { mo. old } \\ \Pi \rightarrow \text { dSPNs }}}{\text { g }}$
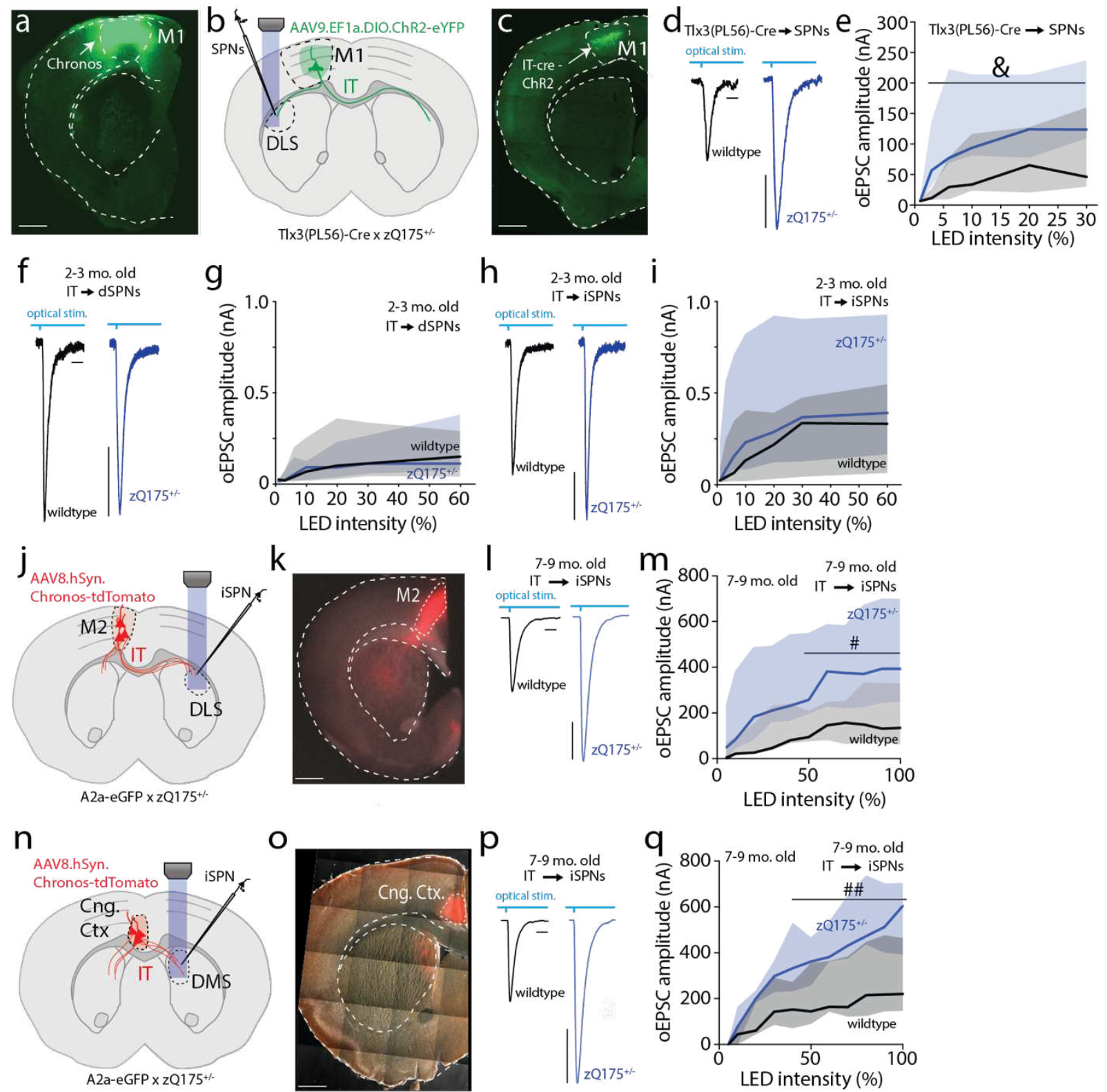

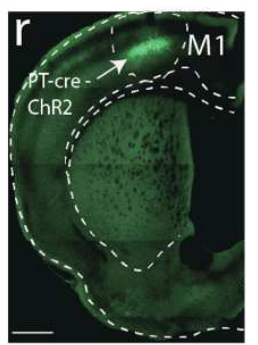

Sim1 KJ18 PT-cre $x$ D2-eGFP x zQ175+/

Figure S1 (a) Confocal image showing AAV9.hSyn.Chronos-GFP expression in M1. (b) Schematics of AAV9.EF1a.DIO.ChR2eYFP injected in primary motor cortex (M1) in a double transgenic mouse obtained crossing Tlx3(PL56)-Cre (expressing Cre in IT neurons) with zQ175 1 - mice. (c) Confocal image showing AAV9.EF1a.DIO.ChR2-eYFP expression in IT neurons in M1. (d) Representative traces of oEPSC obtained by stimulating IT axons and recorded in SPNs (ipsilateral DLS) in 8-9 
month old TIx3(PL56)-Cre $\times$ zQ175/- mice and age-matched wildtype controls. (e) Input/output curves (I/O) in SPNs, wildtype ( $N=2$ mice; $n=5$ neurons) vs. $z Q 175^{+/-}(N=3 ; n=7)$; Significant increase in oEPSCs amplitude in $z Q 175(\&, p=0.031$ in a Wilcoxon Signed Rank Test). (f) Representative traces of oEPSC obtained by stimulating IT axons and recorded in dSPNs

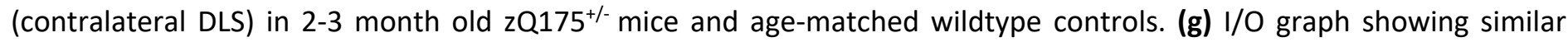
oEPSCs amplitude when plotted against LED intensity in $\mathrm{ZQ} 175^{+/-}$mice compared wildtype. (h) Representative traces of oEPSC, recorded in iSPNs in 2-3 month old mice. (i) l/O graph showing similar oEPSCs amplitude in $z Q 175^{+/-}$mice compared wildtype. (j) Schematics of AAV8.hSyn.Chronos-tdTomato construct stereotaxic injection in secondary motor cortex (M2). (k) Confocal image showing the expression of the chronos construct in M2. (I) Representative traces of oEPSC evoked in iSPNs in contralateral DLS. $(\mathrm{m})$ Graphs showing increased oEPSCs amplitude when plotted against LED intensity in $\mathrm{zQ} 175^{+/-}$mice compared to wildtype. Input/output curves (I/O) in iSPNs (wildtype ( $\mathrm{N}=7$ mice; $\mathrm{n}=7$ neurons) vs. $\mathrm{zQ} 175^{+/-}$ $(\mathrm{N}=7 ; \mathrm{n}=7)$; p-value (LED Intensity, \% of max): n.s. $(5,10,20,30,40 \%)$, \#, significant increase: 0.026 (50\%), 0.026 (60 \%), 0.017 (70\%), 0.017 (80\%), 0.0175 (90\%), 0.0175 (100\%); in a Mann-Whitney non-parametric test). (n) Schematics of AAV8.hSyn.Chronos-tdTomato construct stereotaxic injected in cingulate cortex (Cng. Ctx.). (o) Confocal fluorescence image showing the expression of the chronos construct in Cng. Ctx. (p) Representative traces of oEPSC evoked in iSPNs in contralateral DMS. (q) Graphs showing increased oEPSCs amplitude when plotted against LED intensity in zQ175 ${ }^{+/-}$mice compared control. Input/output curves (I/O) in iSPNs (wildtype ( $N=7$ mice; $n=7$ neurons) vs. $z Q 175^{+/-}(N=7 ; n=7) ; p-v a l u e$ (LED Intensity, \% of max): n.s. (5, 10, 20, 30), \#\#, significant increase: 0.041 (40\%), 0.038 (50\%), 0.017 (60\%), 0.038 (70 $\%), 0.011$ (80\%), 0.007 (90\%), 0.007 (100\%); in a Mann-Whitney non-parametric test). ( $\mathbf{r}$ ) Confocal image showing a coronal hemi-slice expressing AAV9.EF1a.DIO.ChR2-GFP in cortical PT neurons from Sim1 KJ18 PT-cre mice. Scale bars in all confocal images are $1 \mathrm{~mm}$.

Figure S2 - Pancani et al.

a

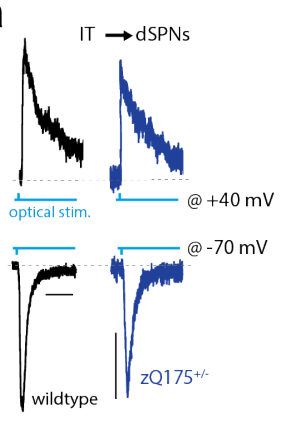

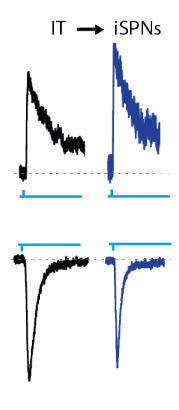

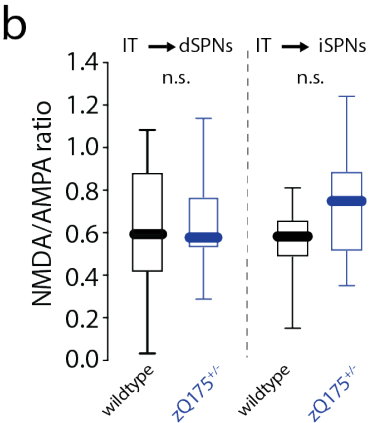

Figure S2 (a) Sample traces depicting synaptic NMDA and AMPA currents optically evoked stimulating IT axons in DLS while holding the cell at $-70 \mathrm{mV}$ (AMPA) or $+40 \mathrm{mV}$ (NMDA). Traces recorded from dSPNs and iSPNs in wildtype (black) compared to $\mathrm{zQ} 175^{+/-}$(blue). Scale bars: $10 \mathrm{~ms}, 50 \mathrm{pA}$. (b) NMDA/AMPA ratios were unchanged in $\mathrm{zQ} 175^{+/-}$ compared to wildtype in both dSPNs and iSPNs. Mann-Whitney non-parametric test (dSPNs wildtype $(N=4, n=11)$,

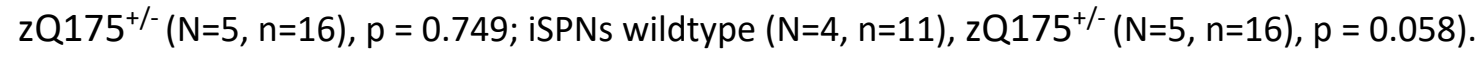

Figure S3 - Pancani et al.

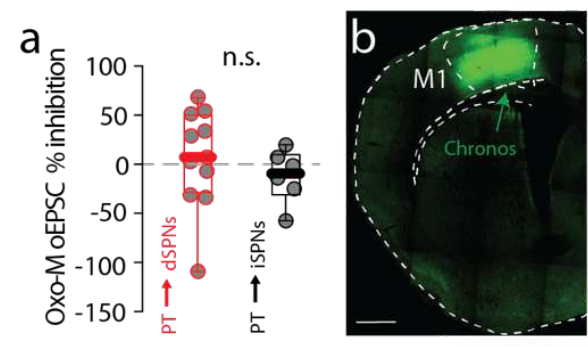

Figure S3 (a) Boxplot summarizing the acute effect of Oxo-M $(10 \mu \mathrm{M})$ on oEPSCs evoked by stimulating PT terminals and monitored during the last 3 min of Oxo-M application in Sim 1 - kj18 PT-cre mice. No different effect was seen in dSPNs 
vs. iSPNs in a Mann-Whitney non-parametric test ( $p=0.3011$; dSPNs $(N=5 ; n=11)$, iSPNs $(N=5, n=6)$. (b) Confocal image showing a coronal hemi-slice expressing AAV9.hSyn.Chronos-GFP in M1 (Scale bar: $1 \mathrm{~mm}$ ).

Figure S4 - Pancani et al.

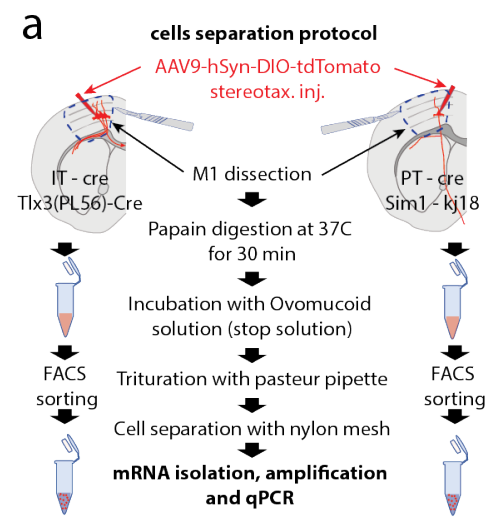

C

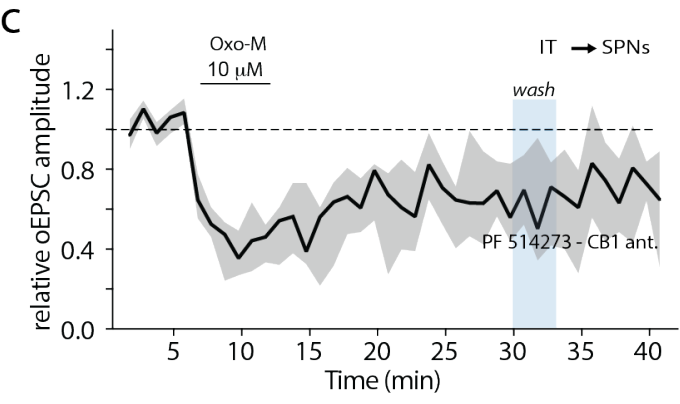

b

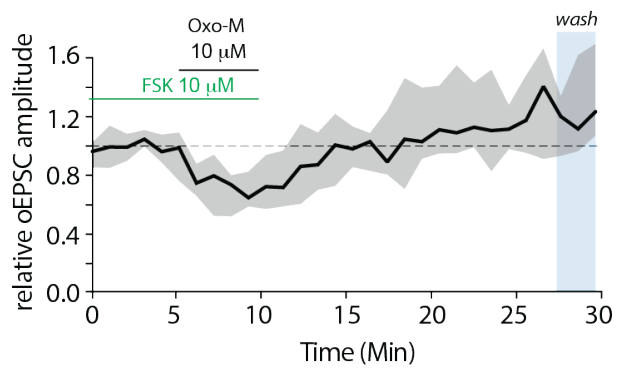

d 100

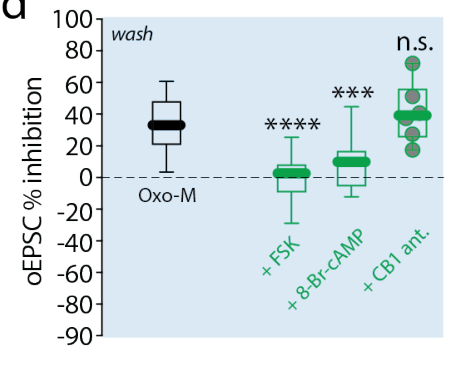

e

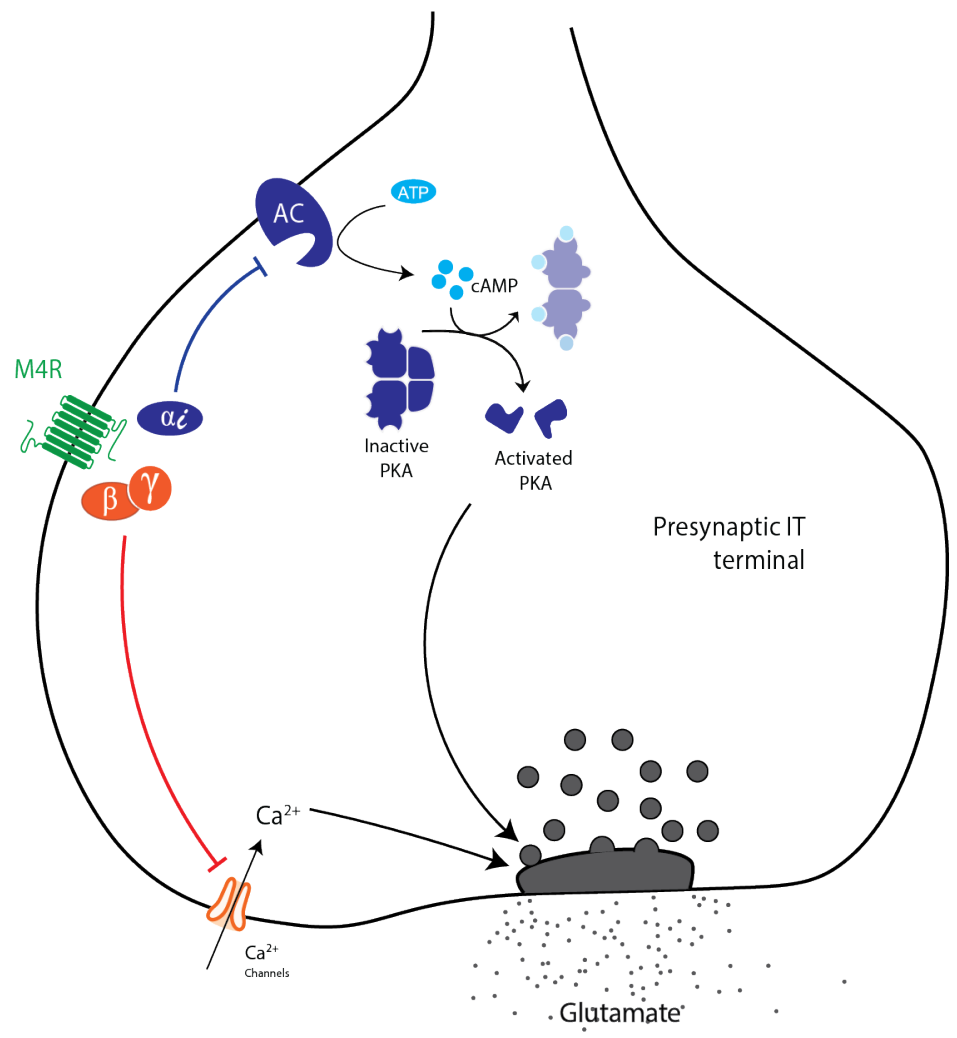

Figure S4 (a) Protocol used to determine the abundance of M2 and M4 mRNA in IT or PT neurons in M1. M1 tissue expressing td tomato in IT (PL56-cre) or PT (Sim 1 - kj18) neurons is removed from freshly cut slices and processed according to protocol (see Materials and Methods). tdTomato positive neurons are enzymatically separated, FACS-sorted, and analyzed. (b) Time-series of oEPSC peak amplitudes normalized to baseline control and plotted over time (median and interquartile range are represented). Oxo-M did not induce LTD at IT-SPNs synapses when slices were pre-incubated with 
forskolin (FSK $10 \mu \mathrm{M}$ ) for 20-30 min before Oxo-M application. (c) pre-incubation with the highly selective CB1 antagonist PF $514273(1 \mu \mathrm{M})$ for 20-30 min. had no effect on the LTD obtained by Oxo-M application. (d) boxplot data summarizing the effect of Oxo-M on oEPSCs during the wash period in slices with and without pre-incubation with FSK, 8-Br-cAMP and PF514273 as described above and in Figure 6. Data group on the left represents the effect measured during Oxo-M application, on the right is the inhibitory effect monitored during the wash period. p-values, Kruskal-Wallis with Dunn's multiple comparison test: Oxo-M ( $N=8, n=22)$ vs. i) Oxo-M + FSK ( $N=5, n=12)$, **** $p=0.0001$; ii) Oxo-M + 8-Br-cAMP (N=4, $\mathrm{n}=10),{ }^{* *} \mathrm{p}=0.0197$; iv) Oxo-M vs. Oxo-M + CB1 ant. ( $\left.\mathrm{N}=2, \mathrm{n}=6\right)$, n.s. $\mathrm{p}>0.9999$. (e) Schematics of a potential mechanism explaining the acute and long-term pre-synaptic inhibition elicited by $M 4$ activation. Activation of $M 4 G_{i / o}$ coupled signaling complement would result in activation of $G_{i \alpha}$ and $G_{i \beta \gamma}$. Our data (Figure $\mathbf{S 4 b}$ and $\mathbf{6 e}$ ) suggest that the LTD generated by mAChR activation is dependent on M4 inhibition of adenylyl cyclase and decrease in PKA activity, since it is eliminated by increasing the levels of cAMP by Forskolin or direct activation of PKA with 8-Br-cAMP both applied before and during the induction phase. Therefore, the long-term effect of Oxo-M would be mediated by $\mathrm{G}_{i \alpha}$. On the other hand, the transient acute inhibition seen with Oxo-M application is not dependent on PKA activity and could be mediated by $\mathrm{Gi}_{\beta \gamma}$ inhibition of voltage gated calcium channels.

Figure S5 - Pancani et al.
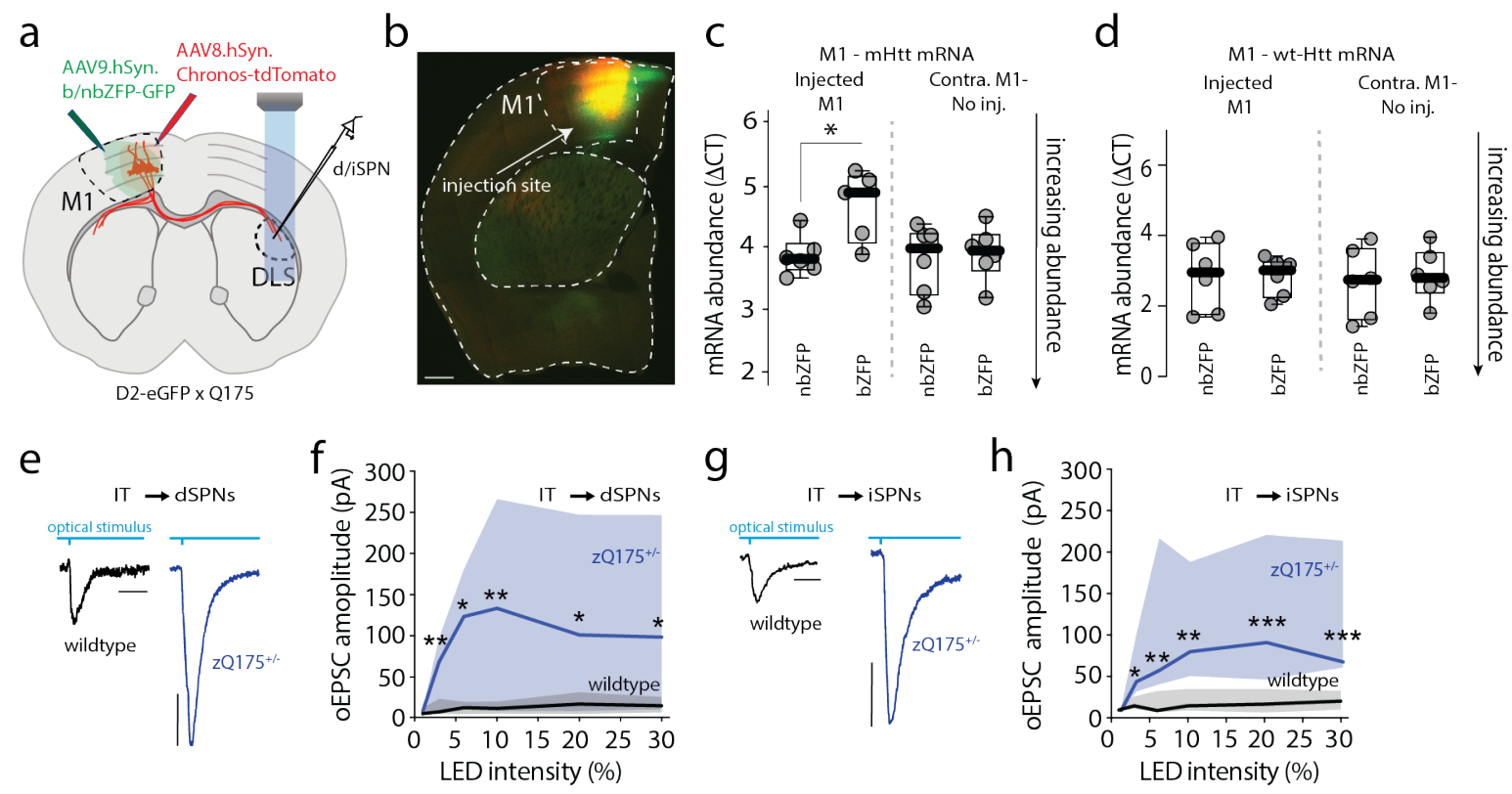

Figure S5 (a) Schematic representation of AAV8.hSyn.Chronos-tdTomato and AAV9.hSyn.bZFP-eGFP constructs being injected in M1. SPNs recording site in the contralateral DLS is also shown. (b) Representative confocal image showing colocalization chronos and ZFP constructs in M1 (scales bar $0.5 \mathrm{~mm}$ ). (c) bZFP expression reduced mHtt mRNA in M1 (evidenced by increased $\Delta C T$ values), compared to the nbZFP-injected mice used as controls $\left({ }^{*} p=0.0303\right.$, Mann-Whitney non-parametric test, $\mathrm{M} 1$ tissue from $\mathrm{N}=5$ animals (bZFP), and $\mathrm{N}=6$ (nbZFP-control), while had no effect on mHtt levels in the contralateral M1 (Contra. M1-No inj., p > 0.05, Mann-Whitney non-parametric test, M1 tissue from N=5 animals (bZFP) and $\mathrm{N}=6$ (nbZFP). (d) bZFP expression had no effect on wildtype-Htt in the injected and contralateral M1 regions. (e-h) ZFP expression in M1 did not normalize corticostriatal IT transmission in dSPNs $(\mathbf{e}, \mathbf{f})$ and iSPNs $(\mathbf{g}, \mathbf{h})$. Shown are the representative traces of oEPSCs elicited by stimulating IT terminals and recorded in dSPNs (e) and iSPNs (g). Graphs summarizing oEPSCs peak amplitude $\mathrm{l} / \mathrm{O}$ data plotted against LED intensity in dSPNs (f; wildtype ( $N=4, \mathrm{n}=11)$ vs. $\mathrm{zQ175}$ ($(\mathrm{N}=5, \mathrm{n}=18), \mathrm{p}$-value (LED Intensity, \% of max): n.s. (1\%), ** 0.0057 (3\%), * 0.0139 (6 \%), ** 0.0078 (10\%), * $0.0207(20$ $\%), 0.0429(30 \%)$, and iSPNs (h; wildtype $(\mathrm{N}=4, \mathrm{n}=10)$ vs. $\mathrm{zQ} 175^{+/-}(\mathrm{N}=5, \mathrm{n}=11), \mathrm{p}$-value (LED Intensity, \% of max): $\mathrm{n} . \mathrm{s}$. $(1 \%), * 0.0357(3 \%), * * 0.0021(6 \%), * * 0.0028(10 \%), * 0.0008(20 \%), * 0.0006(30 \%))$. Mann-Whitney non-parametric tests. 

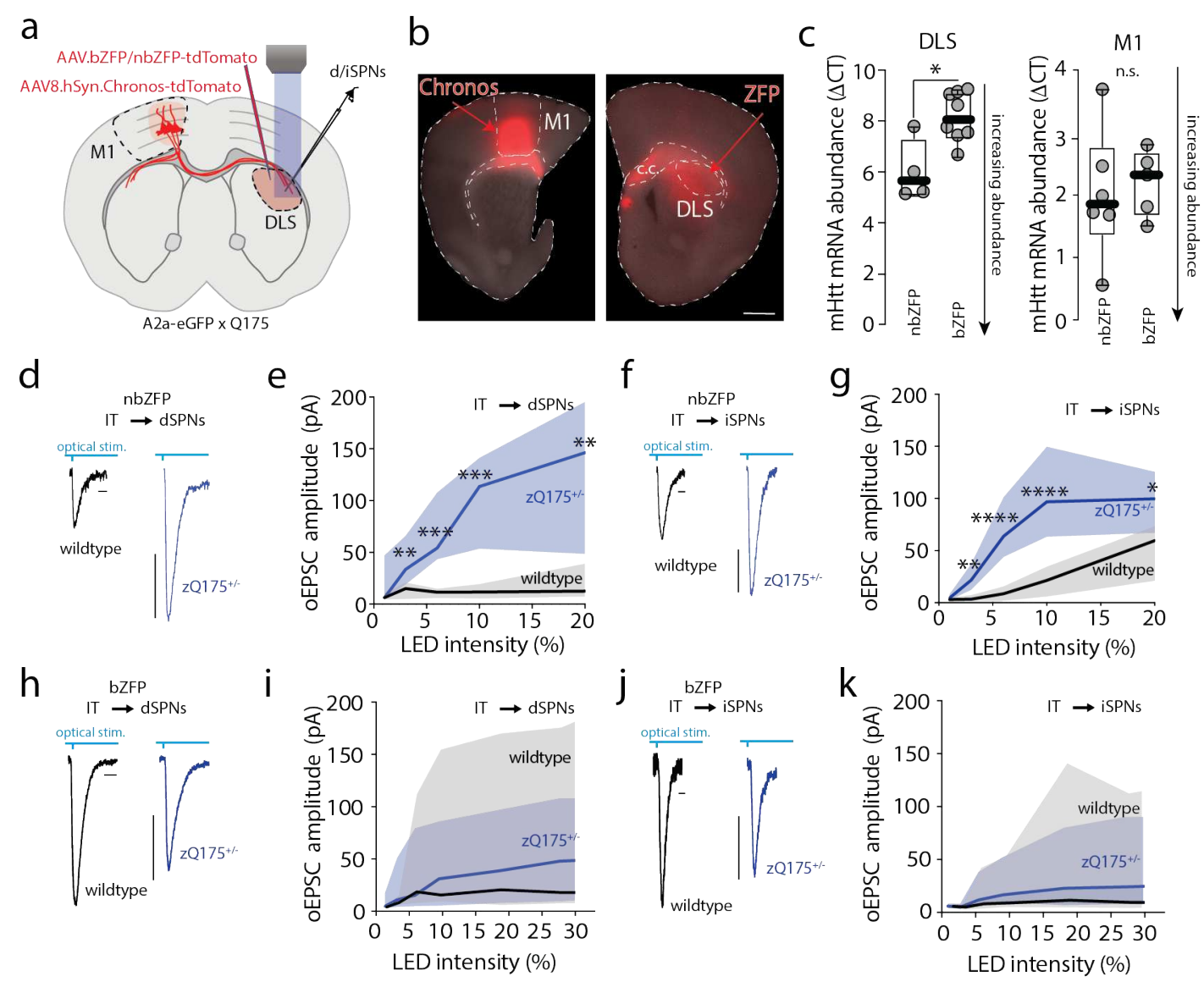

Figure S6 (a) Schematic of AAV8.hSyn.Chronos-TdTomato and AAV.b/nbZFP-tdTomato injected in M1 and contralateral DLS respectively. (b) confocal images of tdTomato fluorescence associated with chronos in M1 (left) and ZFP in contralateral DLS (right; Scale bar: $1 \mathrm{~mm}$ ). (c) bZFP expressed in DLS reduced mHtt mRNA levels compared to nbZFPinjected mice $\left({ }^{*} p=0.0485\right.$ in a Mann-Whitney no-parametric test, $N=4$ mice (bZFP), $N=8$ (nbZFP)), while did not change mHtt mRNA levels in contralateral M1 (n.s., p>0.05 in a Mann-Whitney non-parametric test). (d-g) nbZFP expression in DLS does not normalize IT-SPNs transmission. Representative traces in $\mathbf{d}$ (dSPNs) and $\mathbf{f}$ (iSPNs). Graphs summarizing eEPSCs peak amplitude I/O data plotted against LED intensity in e for dSPNs - wildtype $(N=3, n=6)$ vs. $z Q 175^{+/-}(N=3, n=8), p-$ value (LED Intensity, \% of max): 0.6377 (1\%), 0.0400 (3\%), ***0.0007 (6 \%), ***0.0007 (10\%), ${ }^{* *} 0.0077$ (20\%), - and in g for iSPNs - wildtype $(\mathrm{N}=3, \mathrm{n}=8), \mathrm{zQ} 175^{+/-}(\mathrm{n}=10), \mathrm{p}$-value (Stim. Intensity, \% of max): $0.3254(1 \%), * * 0.0085(3 \%)$, $* * * *<0.0001(6 \%), * * * *<0.0001$ (10\%), 0.0343 (20\%). All tests are Mann-Whitney non-parametric test. (h-k) bZFP construct in DLS normalized IT-SPNs connectivity. Representative traces are shown in $\mathbf{h}$ (dSPNs) and $\mathbf{j}$ (iSPNs). Graphs summarizing eEPSCs peak amplitude $\mathrm{l} / \mathrm{O}$ data plotted against LED intensity are shown in i for dSPNs (wildtype (N=3, $\mathrm{n}=7$ ), zQ175 ${ }^{+-}(\mathrm{N}=6, \mathrm{n}=12)$, p-value (LED Intensity, \% of max): 0.3749 (1\%), 0.5380 (3 \%), >0.9999 (6 \%), 0.9298 (10\%), 0.9298 $(20 \%),>0.9999(30 \%)$ ), and in $\mathbf{k}$ for iSPNs (wildtype $(\mathrm{N}=3, \mathrm{n}=8), \mathrm{zQ175} \mathrm{5}^{+-}(\mathrm{N}=6, \mathrm{n}=13), \mathrm{p}$-value (LED Intensity, \% of max): 0.7108 (1\%), 0.6504 (3 \%), 0.2991 (6 \%), 0.3402 (10\%), 0.9018 (20\%), 0.9017 (30\%)). 

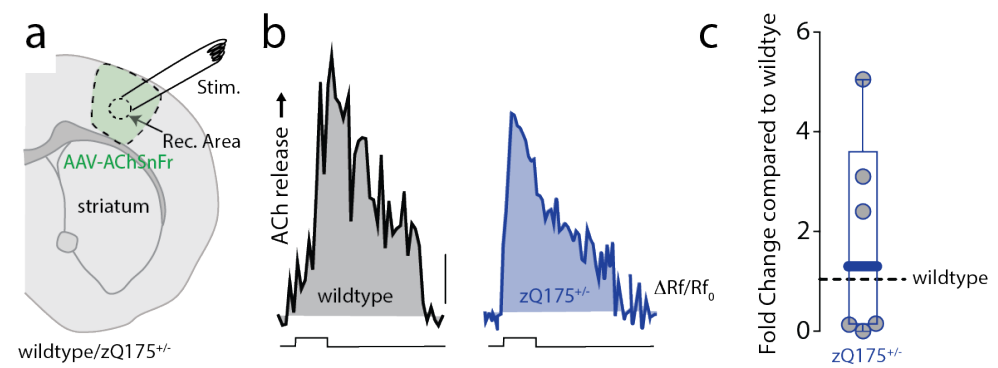

Figure S7 (a) Schematic of AAV.AChSnFr viral construct stereotaxically delivered in M1 of wildtype or zQ175 ${ }^{+/}$mice. (b) Representative traces showing similar evoked ACh released in $\mathrm{zQ}_{175^{+/}}$compared to wildtype. Scale bars: 50 grey values.

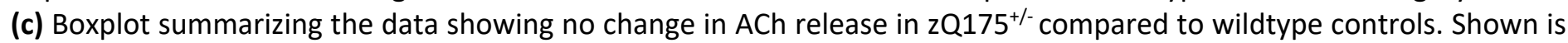
the median from wildtype (dashed line) compared to the fold change in $z Q 175^{+/}$. wildtype ( $N=2$ animals, $n=7$ slices),

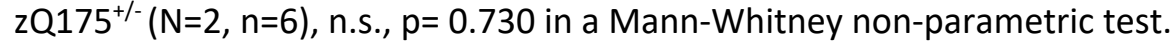

Figure S8 - Pancani et al.
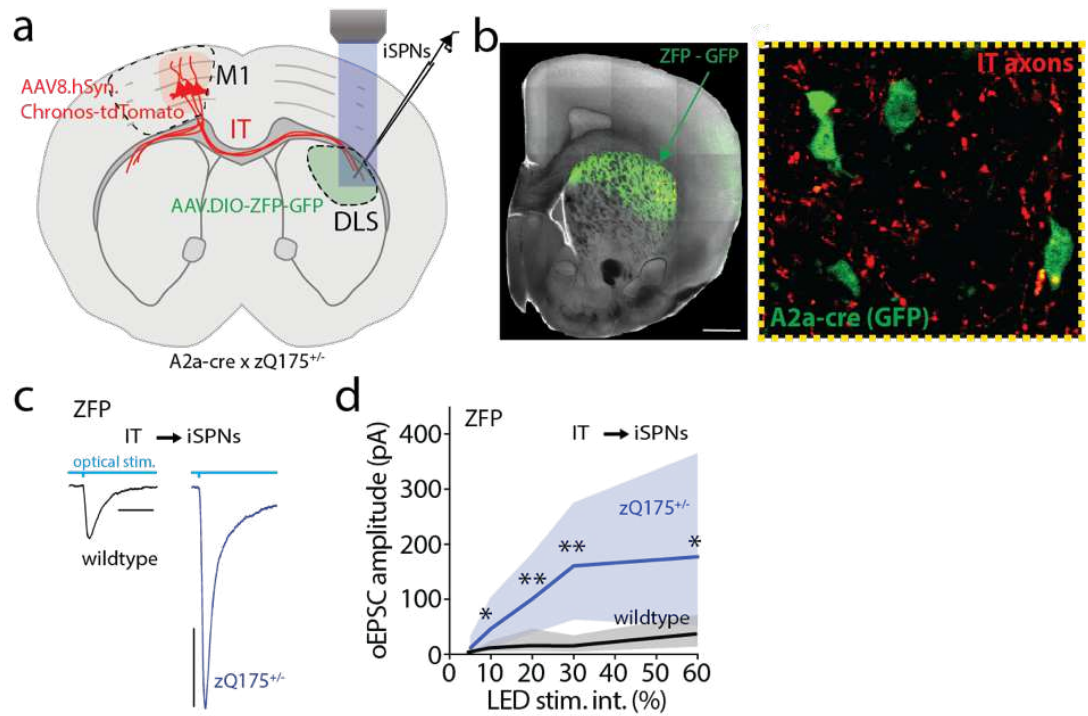

Figure S8 (a) Schematic of AAV8.hSyn.Chronos-TdTomato and AAV.DIO.ZFP-GFP injected in M1 and contralateral DLS respectively, in A2a-cre $x$ zQ175 ${ }^{+/-}$mice. (b) Left, confocal image of a coronal slice expressing AAV.DIO.ZFP-GFP in dorsal striatum (Scale bar: $1 \mathrm{~mm}$ ). Right, closeup of the DLS region evidencing A2A-cre neurons (iSPNs) expressing the ZFP-GFP construct together with tdTomato-positive axons. (c) Representative traces of oEPSC recorded in contralateral DLS obtained by stimulating IT axons. Expression of the cre-dependent ZFP construct specifically in iSPNs did not normalize IT corticostriatal transmission onto iSPNs. Data collected only from iSPNs expressing GFP fluorescence (Scale bar: $10 \mathrm{~ms}$ and 50 pA). (d) Graphs showing increased oEPSCs amplitude when plotted against LED intensity in iSPNs from zQ175 ${ }^{+/-}$mice compared wildtype. I/O relationship, wildtype ( $N=3, n=8$ neurons) vs. $z Q 175^{+/-}(N=3, n=12) ; p$-value (LED Intensity, \% of $\max ):{ }^{*} 0.0124$ (5 \%), *0.0124 (10\%), **0.0096 (20\%), **0.0022 (30\%), *0.0473 (60\%), Mann-Whitney non-parametric tests. 


\section{Methods}

Mice: We used male mice heterozygous for the zQ175 knock-in $\left(\mathrm{zQ}_{175^{+/-}}\right)$with an expanded polyglutamine sequence of $\sim 190$ repeats on a C57/BI6 background B6J.zQ175DN KI mice (Jackson Labs, Stock No. 029928). To study the PT striatal afferents, we used Sim1-kj18-cre mice (MGI:4367070). For the FACS sorting experiments we also used the Tlx3(PL56)-Cre line (MGI:5311700) to investigate IT neurons mRNA expression (http://www.gensat.org/index.html).

Mice were crossed with bacterial artificial chromosome (BAC) D1 dopamine receptor-tdTomato (D1-tdTomato; B6.Cg$\operatorname{Tg}$ (Drd1a-tdTomato)6Calak/J; Jackson Labs, Stock No. 016204) or D2 dopamine receptor-eGFP (D2-eGFP; B6;FVB-Tg(Drd2EGFP/Rpl10a)CP101Htz/J; 030255) lines as specified in the text and figures to identify dSPNs and iSPNs. Both lines have been backcrossed to pure C57/BL6 background. To manipulate cholinergic interneurons activity, we used ChAT-Cre mice

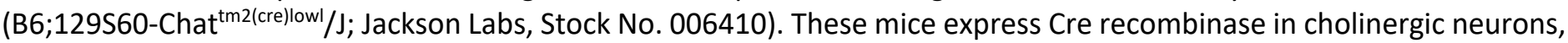
without disrupting endogenous Chat expression. To selectively target iSPNs we also used A2a-Cre mice, a mouse line expressing Cre selectively in iSPNs (Tg(Adora2a-Cre)KG139Gsat; MGI:4361654).

Slice preparation: In accordance with Northwestern University Animal Studies committee, two to three and seven to nine months old mice were deeply anesthetized with ketamine and xylazine, intracardially perfused with ice-cold low-Ca ${ }^{2+}$ artificial cerebrospinal fluid (cutting ACSF) containing $125 \mathrm{mM} \mathrm{NaCl}, 7 \mathrm{mM}$ glucose, $25 \mathrm{mM} \mathrm{NaHCO}, 2.5 \mathrm{mM} \mathrm{KCl}$ and 1.25 $\mathrm{mM} \mathrm{NaH}_{2} \mathrm{PO}_{4}, 0.5 \mathrm{mM} \mathrm{CaCl}_{2}, 2 \mathrm{mM} \mathrm{MgCl}_{2}$ equilibrated with 95\% oxygen and 5\% $\mathrm{CO}_{2}$. Coronal slices, $275 \mu \mathrm{m}$ were cut in cutting ACSF, then incubated at $32{ }^{\circ} \mathrm{C}$ for at least $30 \mathrm{~min}$ in regular ACSF of similar composition to the cutting ACSF but with $2 \mathrm{mM} \mathrm{CaCl}_{2}$ and $1 \mathrm{mM} \mathrm{MgCl}_{2}$. Slice were subsequently stored at R.T. $\left(\sim 25^{\circ} \mathrm{C}\right)$.

Stereotaxic injections: Stereotaxic guided surgeries were performed on mice anesthetized with isoflurane. After positioning the head to obtain a flat skull between bregma and lambda, a small hole was drilled with a micro drill, and a glass pipette was slowly inserted at the following coordinates. Primary motor cortex (M1) injection coordinates were reached using a computer-guided stereotaxic instrument with atlas integration (Angle Two system, Leica) and were (relative to bregma): $1.2 \mathrm{~mm} \mathrm{AP,} 1.6 \mathrm{~mm} \mathrm{ML}$ and $-1.4 \mathrm{~mm}$ DV. For cingulate cortex (Cng. Ctx.): $1.34 \mathrm{~mm} \mathrm{AP,}-0.3 \mathrm{~mm} \mathrm{ML}$ and -1.8 mm DV. For secondary motor cortex (M2): $1.94 \mathrm{~mm} \mathrm{AP,}-0.75 \mathrm{~mm} \mathrm{ML}$ and -1.35 mm DV. For dorsolateral striatum (DLS) coordinates: $1.00 \mathrm{~mm} \mathrm{AP,} 1.83 \mathrm{~mm} \mathrm{ML}$ and -3.04 mm DV.

Electrophysiology: Slices were perfused at 2-3 ml/min with oxygenated regular ACSF containing $2 \mathrm{mM} \mathrm{CaCl} 2$ and $1 \mathrm{mM}$ $\mathrm{MgCl} 2$ at $\sim 25^{\circ} \mathrm{C}$. For all experiments, $20 \mu \mathrm{M}$ Gabazine and $2 \mu \mathrm{M}$ CGP55845 were added to the perfusion media to block $G_{A B A_{A}}$ and $G A B A_{B}$ receptors respectively. SPNs restricted to the dorsolateral striatum were identified using infrared differential interference contrast on an upright Olympus Bx51WI (40X/0.8 NA objective) microscope and a CCD camera connected to a monitor. Somatic whole-cell recordings were made with borosilicate patch pipettes having open tip resistances of 3-5 $\mathrm{M} \Omega$. The intracellular pipette solution contained cesium-based internal solution to reduce the impact of synapses position and maximize voltage control: $115 \mathrm{mM}$ cesium methylsulfonate, $5 \mathrm{mM}$ HEPES, $5 \mathrm{mM}$ tetraethyammonium-Cl, $2 \mathrm{mM}$ QX-314, $0.25 \mathrm{mM}$ EGTA, $2 \mathrm{mM}$ Mg-ATP, $0.5 \mathrm{mM}$ Na-GTP, $10 \mathrm{mM}$ sodium phosphocreatine, $\mathrm{pH} 7.3$ and osmolarity $280-290 \mathrm{mOsm}$. After rupture of the membrane the cells were allowed to dialyze with the internal solution for $15 \mathrm{~min}$. Recordings were obtained using a MultiClamp 700A amplifier and pClamp 10 software. Pipette offset and capacitances were adjusted using MultiClamp 700B Commander software. Data were acquired at $10 \mathrm{kHz}$, filtered at $1 \mathrm{kHz}$ using an 8-pole Bessel filter, and digitized using a DigiData 1322A 16-bit A/D converter. Somatic access resistance was monitored "offline" and "online" by delivering a $200 \mathrm{~ms},-20 \mathrm{mV}$ step in each sweep. Cells were held at -70 mV, not corrected for liquid junction potential. Input/output data from Cingulate cortex (Cng. Ctx.) and M2 (Fig. S1f-m) were obtained using a different setup. The primary difference were object lens, 60X/1.0NA, and the stimulation and display of the electrophysiological recordings that were obtained with freeware WinFluor software (John Dempster, Strathclyde University, Glasgow, UK.).

Patch pipettes (4-6 M $\Omega$ ) were prepared with a Sutter Instruments horizontal puller using borosilicate glass with filament and filled with the internal solution mentioned above. Access resistances were continuously monitored, and experiments were discarded if changes $>20 \%$ were observed. Digitized data were imported for analysis with commercial software (IGOR Pro 6.0, WaveMetrics, Oregon).

Optogenetics: To activate channelrhodopsin (ChR2) or Chronos containing axons in the dorsolateral striatum, we generated $475 \mathrm{~nm} / 30 \mathrm{~mm}$ bandwidth light pulse with a pE 100 LED system (Cool Led) or an X-Cite 110LED Illumination 
System reflected through an eGFP filter cube. The stimulation light passed through a 40x objective lens (or 60x, for the Cng. Ctx. and M2) to produce a $500 \mu \mathrm{m}$ (or $367 \mu \mathrm{m}$ ) diameter column of light at the slice surface. The microscope epiillumination condenser iris on each system was reduced to limit the maximum sample power. The LED power setting was changed manually before stimulation and carefully titrated for physiological response. Unless otherwise specified, blue light pulses ( $3 \mathrm{~ms}$ duration) were triggered through pClamp via TTL. For most of our experiments and to obtain reliable PPR data, we used Chronos rather than ChR2, since ChR2 channels display slow recovery kinetics therefore it was impossible to reach stimulation frequencies necessary for PPR studies without generating a consistent depression of the second EPSC.

Imaging: Large scale montage brain slice images were acquired with the Olympus FV-10i-DUC automated confocal system with 10X/0.4NA (default 2.5 Airy unit pinhole setting) objective lens and higher resolution images with the 60X/1.35NA (default 2 Airy unit pinhole setting) oil-immersion objective. Montage images were acquired with zoom $1(10 \mathrm{x}=1.278 \mathrm{~mm}$ FOV) and $1024 \times 1024$ pixels. The $473 \mathrm{~nm}$ and $559 \mathrm{~nm}$ lasers were used to provide the eGFP and tdTomato images. Dendritic morphology and spine location identification as well as dynamic imaging was performed with Prairie Technologies Ultima series 2-photon excitation microscope system. The system foundation was an Olympus Bx51WI microscope equipped with a $60 x / 1.0 N A(2 \mathrm{~mm}$ WD) water-dipping objective lens. Stage, lens focus, and manipulator remote motion control provided by Luigs and Neumann FM-380 plus SM-7 controllers. 2-photon excitation laser was Chameleon Ultra1 (690nm to $1040 \mathrm{~nm}$ ) and SCRACM stimulation via Coherent OBIS FP473-LX laser (50mW post-fiber). Dual channel green and red non-de-scanned detection provided by green $(490 \mathrm{~nm}$ to $560 \mathrm{~nm}) \mathrm{GaAsP}$ cathode PMT (H10770B, Hamamatsu) and red ( $580 \mathrm{~nm}$ to $625 \mathrm{~nm}$ ) by multi-alkali cathode PMT (R3986, Hamamatsu). The frame scan parameters were typically set at 256 $\mathrm{x} 256 \mathrm{pixel} / \mathrm{image}$ with a frame period of $\sim 0.500 \mathrm{~s}$.

Subcellular Channelrhodopsin-2-assisted circuit mapping (sCRACM): after whole-cell configuration was reached, cell was dialyzed for at least 15-20 min with an internal solution containing Alexa Fluor $568(25 \mu \mathrm{M})$. sCRACM experiments were performed in the presence of $\Pi \mathrm{TX} 1 \mu \mathrm{M}, 4-\mathrm{AP} 10 \mu \mathrm{M}$ to block propagated activity, along with $\mathrm{GABA}_{\mathrm{A} / \mathrm{B}}$ antagonists. Fluorescence images were acquired with a 2-photon laser scanning microscopy (2PLSM) setup as described above with $780 \mathrm{~nm}$ laser wavelength and red emission channel. Dendritic branches more than $\sim 80 \mu \mathrm{m}$ from the soma ("distal") were selected only after ensuring that no other fluorescent dendrites of the patched cell were present above or below the branch tested. In each round of stimulations, each parfocal spine was stimulated with 1 ms-long laser pulse (i.s.i. $2 \mathrm{~s}$, Coherent Obis Laser $473 \mathrm{~nm}$ ) delivering $~ 0.36 \mathrm{~mW}$ after 60x/1.0NA objective lens. Parfocal dendritic branches where more than $70 \%$ of the spines responded were excluded to reduce false positives. The results of three stimulation rounds (the sequence interval was approximately 1 minute) were averaged and included in the study. The interpretation of the proximal dendrites data was more problematic due to constraints relative to the SCRACM technique and the radial shape of the SPNs dendritic branches.

Fluorescent imaging of AChSnFr: Acetylcholine release was evoked in acute slices by local electrical stimulation in DLS using a bipolar electrode (Micro Probes WE3ST32.0B10) and a Digitimer DS3 stimulator. Stimulator control was $1 \mathrm{~mA}, 1 \mathrm{~ms}$ x 20 pulses at $2 \mathrm{~Hz}$ (9.5s long). The external ACSF was supplemented with Gabazine $10 \mu \mathrm{M}$ and CGP54626 $10 \mu \mathrm{M}$ to eliminate GABAergic activity. Fluorescence images were acquired with a 2PLSM setup as described above with $950 \mathrm{~nm}$ laser wavelength and green detection channel. The probe's fluorescence values were obtained by averaging the grey values in a circular region of interest (ROI, about $100 \mu \mathrm{m}$ in diameter, See Fig. 3b) in each frame, using the Prairie brightness over time (BOT) function or Image $\mathrm{J}(\mathrm{NIH})$, and a time-lapse of the fluorescence changes was built for each experiment. Each trace was normalized to the minimum fluorescence $\left(F_{\min }\right)$ obtained by perfusing $\mathrm{Cd}^{2+} 2 \mathrm{mM}$ to block vesicular release, and a and maximum $\left(F_{\max }\right)$ obtained perfusing $A C h 100 \mu \mathrm{M}$ at the end of each experiment to obtain a Normalized Fluorescence trace. The formula used was: Normalized Fluorescence $\left(F_{n}\right)=\left(F-F_{\min }\right) /\left(F_{\max }-F_{\min }\right)$. $F_{n}$ traces were used to calculate the dynamic response of the acetylcholine transient $\left[\left(F_{n}-F_{0}\right) *\right.$ time $\left.(s)\right]$. Each dynamic response data point was calculated from the median of at least two-three ROIs/slice. $\mathrm{Cd}^{2+}$ did not have a significant effect on baseline fluorescence, indicating that tonic ACh release is low in the ex vivo slices. The experiments in Supplementary Figure 7 were not normalized traces, but instead the simple $\Delta f / f_{0}$ where $f_{0}$ value did have no-laser background subtraction applied.

Quantitative real-time PCR analysis of M2/M4 mRNA Expression: Experimental procedures and data processing for fluorescence-activated cell sorting (FACS) were as previously described with minor modifications ${ }^{1}$. We used PT-cre or IT- 
cre mice injected with an AAV-DIO-tdTomato virus. Thirty days post injection primary cortex tissue was isolated from 300 $\mu \mathrm{m}$ thick coronal slices and single-cell suspensions were generated using a combination of enzymatic and mechanical dissociation procedures (Fig. S4a). PT or IT neurons were separated on a cell sorter (BD FACSAria SORP system and BD FACSymphony S6 SORP system, purchased through the support of NIH 1S10OD011996-01 and 1S100D026814-01), based on tdTomato fluorescence. Approximately 3000-7000 cells from cortical tissue collected from 2 mice/sample. Total RNA was isolated from each PT and IT sample with the RNeasy micro kit (Qiagen) as previously described (Gao et al., 2013). Due to the relatively low cell count in the samples, cRNA was amplified using Ambion WT expression kit (Life Technologies). Five hundred or less ng of total RNA was copied to cDNA using the QuantiTect Reverse Transcription Kit (Qiagen) in a final volume of $20 \mu \mathrm{l}$. Real-time quantitative PCR reactions were performed in a 7500 Fast Real Time PCR System (Life Technologies). PCR reactions were performed in duplicates in a total volume of $20 \mu$ l containing 1-2.5 $\mu$ of cDNA solution and $1 \mu$ l of Taqman probe of the specific gene (Life Technologies). GAPDH was also estimated in each sample to normalize the amount of total RNA (or cRNA) used to perform relative quantifications. Fold change compared to GAPDH was expressed in relative units (r.u). The assay identifications used were: Chrm2; muscarinic receptor 2: Mm01701855_s1; Chrm4; muscarinic receptor4: Mm00432514_s1.

Quantitative real-time PCR analysis of Htt mRNA Expression: RNA was isolated using RNAeasy kit (Qiagen) from striatal and cortical tissue of mice injected with ZFP AAV. The RNA was reverse transcribed with Superscript IV VILO Master Mix (Thermo Fisher Scientific). Quantitative real-time PCR was performed using an ABI StepOnePlus Real Time PCR system with TaqMan Fast Edvanced Master Mix (Thermo Fisher Scientific). The relative abundance of different transcripts was assessed by quantitative PCR in triplicate. The following primers/TaqMan probes (Thermo Fisher Scientific) were used for PCR amplification: Hprt: Mm03024075_m1; wild-type-mouse Htt_fw: CAGGTCCGGCAGAGGAAC, Mut-mouseHtt_Q175_fw: GCCCGGCTGTGGCTGA, Mut and wild-type Htt_rv*: TTCACACGGTCTTTCTTGGTGG (*wild-type and mutant $\mathrm{Htt}$ share the same reverse primer sequence), probe: TGCACCGACCAAAGAAGGAACTCTCA . Experimental Ct values were normalized to Hprt values using the formula: $\Delta \mathrm{Ct}=\mathrm{CtHtt}-\mathrm{Ct}$ (Hprt). The final expression levels were shown as $\Delta \mathrm{Ct}$ values.

Vectors and drugs: AAV9.Syn.Chronos-GFP.WPRE.bGH (Addgene 59170, $2.23 \times 10^{13} \mathrm{gc} / \mathrm{ml}$ ), AAV9.EF1a.DIO.hChR2 (H134R)-eYFP.WPRE.hGH (Addgene 20298, $2.11 \times 10^{13} \mathrm{gc} / \mathrm{ml}$ ) and AAV9.CAG.Flex.tdTomato.WPRE.bGH (Addgene 28306, $2.51 \times 10^{13} \mathrm{gc} / \mathrm{ml}$ ), AAV9-Syn-DIO-rev-PSAM-L141F-Y115F-5HT3HC-IRES-GFP-WPRE (PSAM-5HT, $\left.2.04 \times 10^{13} \mathrm{gc} / \mathrm{ml}\right)$. were supplied by University of Pennsylvania Vector Core and used for contralateral IT recordings, ipsilateral PT recordings. Sangamo Bioscience zinc finger repressor protein plasmids ZFP-30645 (mHtt repressing ZFP; bZFP), and DNA binding deleted control plasmid ZFP-DBD (nbZFP) were obtained via CHDI Foundation, recloned in pFastbac ( $p F B$ ) AAV shuttle vectors to produce hSyn-NLS-ZFP-30645-2A-eGFP-WPRE-bGHpA, hSyn-NLS-ZFP-deltaDBD-2A-eGFP-WPRE-bGHpA, CMVNLS-ZFP-30645-2A-tdTomato-Sv40pA, CMV-NLS-ZFP-deltaDBD-2A-tdTomato-SV40pA, and the Cre-dependent variants hSyn-DIO-NLS-ZFP-30645-2A-EGFP-WPRE-bGHpA and hSyn-DIO-NLS-ZFP-deltaDBD-2A-EGFP-WPRE-bGHpA, and packaged into AAV9 by Virovek": AAV9.hSyn.ZFP30645.Flag.EGFP.WPRE (bZFP; $2.11 \quad \mathrm{x} \quad 10^{13} \mathrm{vg} / \mathrm{ml}$ ), AAV9.hSyn.ZFP.control.Flag.2A.GFP.WPRE (nbZFP; $2.06 \times 10^{13} \mathrm{vg} / \mathrm{ml}$ ), AAV9.CMV.ZFP30645.Flag.TdTomato.WPRE (bZFP; $2.28 \times 10^{13} \mathrm{vg} / \mathrm{ml}$ ), AAV9.CMV.ZFP.control.Flag.TdTomato.WPRE (nbZFP; $2.03 \times 10^{13} \mathrm{vg} / \mathrm{ml}$ ), and the cre-dependent variants: AAV9.hSyn.DIO.ZFP.control.2A.TdTomato $\quad$ (DIO.nbZFP; $\quad 2.12 \quad x \quad 10^{13} \quad \mathrm{vg} / \mathrm{ml}$ ), AAV9.hSyn.DIO.ZFP.30645.2A.TdTomato (DIO.bZFP; $2.13 \times 10^{13} \mathrm{vg} / \mathrm{ml}$ ). All drugs were obtained from Hello Bio, Sigma or Tocris. PSEM ${ }^{895}$ was obtained from S.M. Sternson (Howard Hughes Medical Institute) and Apex Scientific. Acetylcholine sniffers AAVs (AChSnFr; pAAV1/2-hSynap.iAChSnFR.X513 V9; $0.38 \times 10^{13} \mathrm{gc} / \mathrm{ml}$ ) were kindly donated by Loren Looger at Janelia.

Data analysis. pClamp data files were analyzed using Clampfit (Molecular Devices) and Excel (Microsoft). ACh release data were also analyzed with Clampfit, Excel or a custom Matlab routine. Graphs and figures were assembled with GraphPad Prism (GraphPad software) and Illustrator (Adobe). All Boxplots represent median and interquartile range, whiskers $\mathrm{min} / \mathrm{max}$ values. All data are reported as median except when specified otherwise. Statistical analysis was performed in GraphPad Prism, using nonparametric statistic tests unless specified otherwise.

\section{Bibliography}


Plotkin, J. L. et al. Regulation of dendritic calcium release in striatal spiny projection neurons. J Neurophysiol 110, 2325-2336, doi:10.1152/jn.00422.2013 (2013). 